\title{
Fourier coefficients of a class of ETA quotients of weight 20 with level 12
}

\author{
Barış Kendirli \\ Department of Mathematics, Istanbul Aydın University, Turkey \\ Email: bariskendirli@aydin.edu.tr
}

Copyright (C)2015 Barış Kendirli. This is an open access article distributed under the Creative Commons Attribution License, which permits unrestricted use, distribution, and reproduction in any medium, provided the original work is properly cited.

\section{Abstract}

Williams[18] and later Yao, Xia and Jin[15] discovered explicit formulas for the coefficients of the Fourier series expansions of a class of eta quotients. Williams expressed all coefficients of 126 eta quotients in terms of $\sigma(n), \sigma\left(\frac{n}{2}\right), \sigma\left(\frac{n}{3}\right)$ and $\sigma\left(\frac{n}{6}\right)$ and Yao, Xia and Jin, following the method of proof of Williams, expressed only even coefficients of 104 eta quotients in terms of $\sigma_{3}(n), \sigma_{3}\left(\frac{n}{2}\right), \sigma_{3}\left(\frac{n}{3}\right)$ and $\sigma_{3}\left(\frac{n}{6}\right)$.Here, we will express the even Fourier coefficients of 570 eta quotients in terms of $\sigma_{19}(n), \sigma_{19}\left(\frac{n}{2}\right), \sigma_{19}\left(\frac{n}{3}\right), \sigma_{19}\left(\frac{n}{4}\right), \sigma_{19}\left(\frac{n}{6}\right)$ and $\sigma_{19}\left(\frac{n}{12}\right)$.

Keywords: Dedekind eta function; eta quotients; Fourier series.

\section{Introduction}

The divisor function $\sigma_{i}(n)$ is defined for a positive integer $i$ by

$\sigma_{i}(n):=\sum_{d \text { positive integer }, d \mid n} d^{i}$, if $n$ is a positive integer, and

$\sigma_{i}(n):=0$, if $n$ is not a positive integer.

The Dedekind eta function is defined by

$\eta(z):=q^{1 / 24} \prod_{n=1}^{\infty}\left(1-q^{n}\right)$,

where

$q:=e^{2 \pi i z}, z \in H=\{x+i y: y>0\}$

and an eta quotient of level $n$ is defined by

$f(z):=\prod_{m \mid n} \eta(m z)^{a_{m}}, n, m \in \mathbb{N}, a_{m} \in Z$.

It is interesting and important to determine explicit formulas of the Fourier coefficients of eta quotients, because they are the building blocks of modular forms of level $\mathrm{n}$ and weight $\mathrm{k}$. The book of Köhler [13] (Chapter 3, pg.39) describes such expansions by means of Hecke Theta series and develops algorithms for the determination of suitable 
eta quotients. One can find more information in [3], [6], [14], [16], [17]. I have determined the Fourier coefficients of the theta series associated to some quadratic forms, see [7], [8], [9][10], [11] and [12].

Recently, Williams, see [18] discovered explicit formulas for the coefficients of Fourier series expansions of a class of 126 eta quotients in terms of $\sigma(n), \sigma\left(\frac{n}{2}\right), \sigma\left(\frac{n}{3}\right)$ and $\sigma\left(\frac{n}{6}\right)$. One example is as follows:

$\frac{\eta^{2}(2 z) \eta^{4}(4 z) \eta^{6}(6 z)}{\eta^{2}(z) \eta^{2}(3 z) \eta^{4}(12 z)}=1+\sum_{n=1}^{\infty} c(n) q^{n}$

where

$c(n)=2 \sigma(n)-3 \sigma(n / 2)+4 \sigma(n / 4)+9 \sigma(n / 6)-36 \sigma(n / 12)$.

Then Yao, Xia and Jin [15] expressed the even Fourier coefficients of 104 eta quotients in terms of $\sigma_{3}(n), \sigma_{3}\left(\frac{n}{2}\right), \sigma_{3}\left(\frac{n}{3}\right)$ and $\sigma_{3}\left(\frac{n}{6}\right)$. One example is as follows:

$\frac{\eta^{25}(2 z) \eta^{4}(3 z)}{\eta^{12}(z) \eta^{5}(4 z) \eta^{3}(6 z) \eta(12 z)}=1+\sum_{n=1}^{\infty} c(n) q^{n}$

where

$c(2 n)=65 \sigma_{3}(n)-68 \sigma_{3}(n / 2)-81 \sigma_{3}(n / 3)+324 \sigma_{3}(n / 6)$.

After that we find that we can express the even Fourier coefficients of 570 eta quotients in terms of

$\sigma_{19}(n), \sigma_{19}\left(\frac{n}{2}\right), \sigma_{19}\left(\frac{n}{3}\right), \sigma_{19}\left(\frac{n}{4}\right), \sigma_{19}\left(\frac{n}{6}\right)$ and $\sigma_{17}\left(\frac{n}{12}\right)$,see Table 3. One example is as follows:

$\frac{\eta^{36}(2 z) \eta^{38}(12 z)}{\eta^{18}(4 z) \eta^{16}(6 z)}=1+\sum_{n=1}^{\infty} c(n) q^{n}$

where

$c(2 n)=-\frac{1}{7971615} \sigma_{19}(2 n)+\frac{174763}{23914845} \sigma_{19}(n)-\frac{524288}{71744535} \sigma_{19}(n / 2)$.

We also see that the odd Fourier coefficients of 1208 eta quotients are zero and even coefficients can be expressed by simple formula. Now let

$$
\begin{aligned}
& f_{1}:=\sum_{n=0}^{\infty} f_{1}(n) q^{n}=\frac{\eta^{43}(4 z) \eta^{43}(6 z)}{\eta^{29}(2 z) \eta^{17}(12 z)} \\
& f_{2}=\sum_{n=0}^{\infty} f_{2}(n) q^{n}=\frac{\eta^{38}(4 z) \eta^{48}(6 z)}{\eta^{28}(2 z) \eta^{18}(12 z)} \\
& f_{3}=\sum_{n=0}^{\infty} f_{3}(n) q^{n}=\frac{\eta^{50}(4 z) \eta^{24}(6 z)}{\eta^{28}(2 z) \eta^{6}(12 z)} \\
& f_{4}=\sum_{n=0}^{\infty} f_{4}(n) q^{n}=\frac{\eta^{50}(4 z) \eta^{48}(6 z)}{\eta^{28}(2 z) \eta^{30}(12 z)} \\
& f_{5}=\sum_{n=0}^{\infty} f_{5}(n) q^{n}=\frac{\eta^{45}(4 z) \eta^{29}(6 z)}{\eta^{27}(2 z) \eta^{7}(12 z)}
\end{aligned}
$$




$$
\begin{aligned}
& f_{6}=\sum_{n=0}^{\infty} f_{6}(n) q^{n}=\frac{\eta^{40}(4 z) \eta^{34}(6 z)}{\eta^{26}(2 z) \eta^{8}(12 z)} \\
& f_{7}=\sum_{n=0}^{\infty} f_{7}(n) q^{n}=\frac{\eta^{44}(4 z) \eta^{36}(12 z)}{\eta^{22}(2 z) \eta^{18}(6 z)}, \\
& f_{8}=\sum_{n=0}^{\infty} f_{8}(n) q^{n}=\frac{\eta^{47}(4 z) \eta^{15}(6 z) \eta^{3}(12 z)}{\eta^{25}(2 z)}, \\
& f_{9}=\sum_{n=0}^{\infty} f_{9}(n) q^{n}=\frac{\eta^{47}(4 z) \eta^{39}(6 z)}{\eta^{25}(2 z) \eta^{21}(12 z)}, \\
& f_{10}=\sum_{n=0}^{\infty} f_{10}(n) q^{n}=\frac{\eta^{30}(4 z) \eta^{44}(6 z)}{\eta^{24}(2 z) \eta^{10}(12 z)}, \\
& f_{11}=\sum_{n=0}^{\infty} f_{11}(n) q^{n}=\frac{\eta^{44}(4 z) \eta^{6}(6 z) \eta^{12}(12 z)}{\eta^{22}(2 z)}, \\
& f_{12}=\sum_{n=0}^{\infty} f_{12}(n) q^{n}=\frac{\eta^{15}(2 z) \eta^{35}(4 z) \eta^{11}(12 z)}{\eta^{21}(6 z)}, \\
& f_{13}=\sum_{n=0}^{\infty} f_{13}(n) q^{n}=\frac{\eta^{25}(4 z) \eta^{49}(6 z)}{\eta^{23}(2 z) \eta^{11}(12 z)}, \\
& f_{14}=\sum_{n=0}^{\infty} f_{14}(n) q^{n}=\frac{\eta^{27}(4 z) \eta^{11}(6 z) \eta^{23}(12 z)}{\eta^{21}(2 z)}, \\
& f_{15}=\sum_{n=0}^{\infty} f_{15}(n) q^{n}=\frac{\eta^{5}(4 z) \eta^{45}(6 z) \eta^{9}(12 z)}{\eta^{19}(2 z)} \\
& f_{16}=\sum_{n=0}^{\infty} f_{16}(n) q^{n}=\frac{\eta^{49}(4 z) \eta(6 z) \eta^{13}(12 z)}{\eta^{23}(2 z)} \\
& f_{17}=\sum_{n=0}^{\infty} f_{17}(n) q^{n}=\frac{\eta^{43}(4 z) \eta^{31}(12 z)}{\eta^{17}(2 z) \eta^{17}(6 z)}, \\
& f_{18}=\sum_{n=0}^{\infty} f_{18}(n) q^{n}=\frac{\eta^{50}(6 z) \eta^{8}(12 z)}{\eta^{18}(2 z)}, \\
& f_{19}=\sum_{n=0}^{\infty} f_{19}(n) q^{n}=\frac{\eta^{12}(4 z) \eta^{20}(6 z) \eta^{20}(12 z)}{\eta^{12}(2 z)}, \\
& f_{20}=\sum_{n=0}^{\infty} f_{20}(n) q^{n}=\frac{\eta^{16}(4 z) \eta^{16}(6 z) \eta^{16}(12 z)}{\eta^{8}(2 z)}, \\
& f_{21}=\sum_{n=0}^{\infty} f_{21}(n) q^{n}=\frac{\eta^{20}(4 z) \eta^{12}(6 z) \eta^{12}(12 z)}{\eta^{4}(2 z)}, \\
& f_{22}=\sum_{n=0}^{\infty} f_{22}(n) q^{n}=\frac{\eta^{15}(4 z) \eta^{17}(6 z) \eta^{11}(12 z)}{\eta^{3}(2 z)}, \\
& f_{23}=\sum_{n=0}^{\infty} f_{23}(n) q^{n}=\frac{\eta^{11}(2 z) \eta^{17}(4 z) \eta^{15}(6 z)}{\eta^{3}(12 z)}, \\
& f_{24}=\sum_{n=0}^{\infty} f_{24}(n) q^{n}=\eta(2 z) \eta^{7}(4 z) \eta^{13}(6 z) \eta^{19}(12 z) \text {, }
\end{aligned}
$$




$$
\begin{aligned}
& f_{25}=\sum_{n=0}^{\infty} f_{25}(n) q^{n}=\eta(2 z) \eta^{19}(4 z) \eta^{13}(6 z) \eta^{7}(12 z), \\
& f_{26}= \sum_{n=0}^{\infty} f_{26}(n) q^{n}=\eta^{2}(2 z) \eta^{2}(4 z) \eta^{18}(6 z) \eta^{18}(12 z), \\
& f_{27}= \sum_{n=0}^{\infty} f_{27}(n) q^{n}=\frac{\eta^{12}(2 z) \eta^{20}(6 z) \eta^{20}(12 z)}{\eta^{12}(4 z)}, \\
& f_{28}= \sum_{n=0}^{\infty} f_{28}(n) q^{n}=\eta^{4}(2 z) \eta^{16}(4 z) \eta^{4}(6 z) \eta^{16}(12 z), \\
& f_{29}= \sum_{n=0}^{\infty} f_{29}(n) q^{n}=\frac{\eta^{16}(2 z) \eta^{16}(6 z) \eta^{16}(12 z)}{\eta^{8}(4 z)} \\
& f_{30}= \sum_{n=0}^{\infty} f_{30}(n) q^{n}=\frac{\eta^{16}(2 z) \eta^{16}(4 z) \eta^{16}(12 z)}{\eta^{8}(6 z)}, \\
& f_{31}= \sum_{n=0}^{\infty} f_{31}(n) q^{n}=\eta^{6}(2 z) \eta^{18}(4 z) \eta^{14}(6 z) \eta^{2}(12 z), \\
& f_{32}= \sum_{n=0}^{\infty} f_{32}(n) q^{n}=\frac{\eta^{16}(2 z) \eta^{16}(4 z) \eta^{16}(6 z)}{\eta^{8}(12 z)}, \\
& f_{33}= \sum_{n=0}^{\infty} f_{33}(n) q^{n}=\frac{\eta^{20}(2 z) \eta^{20}(4 z) \eta^{12}(12 z)}{\eta^{12}(6 z)}, \\
& f_{34}= \sum_{n=0}^{\infty} f_{34}(n) q^{n}=\frac{\eta^{20}(2 z) \eta^{20}(4 z) \eta^{12}(6 z)}{\eta^{12}(12 z)}, \\
& f_{n=0}^{\infty} f_{35}(n) q^{n}=\frac{\eta^{30}(2 z) \eta^{30}(4 z)}{\eta^{10}(6 z) \eta^{10}(12 z)}
\end{aligned}
$$

The proof of the following Lemma about these coefficients is obvious.

Lemma 1.1 For $n=1,2, \ldots$,

$f_{1}(2 n)=\cdots=f_{18}(2 n)=0$,

$f_{19}(2 n-1)=\cdots=f_{35}(2 n-1)=0$.

\section{Main results}

Now we can state our main Theorem:

Theorem 2.1 Let $b_{1}, b_{2}, \cdots, b_{5}$ be non-negative integers satisfying

$b_{1}+b_{2}+\cdots+b_{5} \leq 40$.

Define the integers $a_{1}, a_{2}, a_{3}, a_{4}, a_{6}, a_{12}$ by

$a_{1}:=-b_{1}+2 b_{2}-2 b_{3}-4 b_{4}-b_{5}+40$,

$a_{2}:=3 b_{1}+b_{2}+3 b_{3}+10 b_{4}+b_{5}-100$, 
$a_{3}:=3 b_{1}+2 b_{2}+6 b_{3}+4 b_{4}+3 b_{5}-120$,

$a_{4}:=-2 b_{1}-b_{2}-b_{3}-4 b_{4}+2 b_{5}+40$,

$a_{6}:=-9 b_{1}-7 b_{2}-9 b_{3}-10 b_{4}-7 b_{5}+300$,

$a_{12}:=6 b_{1}+3 b_{2}+3 b_{3}+4 b_{4}+2 b_{5}-120$.

Now define the rational numbers $\left\{k_{i}: i=0, \ldots, 40\right\}$ by

$\frac{1}{2^{b_{1}+b_{5}}} x^{b_{1}}(1-x)^{b_{2}}(1+x)^{b_{3}}(1+2 x)^{b_{4}}(2+x)^{b_{5}}=\sum_{i=0}^{40} k_{i} x^{i}$.

Define the rational numbers

$c_{1}, c_{2}, c_{3}, c_{4}, c_{6}, c_{12}, r_{1}, r_{2}, \ldots, r_{34}$

and $r_{35}$ as in www.bariskendirli.com.tr/weight20/Table 1. Here $\left\{f_{1}, \ldots f_{35}\right\} \backslash\left\{f_{7}, f_{12}, f_{14}, f_{15}, f_{17}, f_{18}, f_{35}\right\} \in S_{20}\left(\Gamma_{0}(12)\right)$, $f_{7}, f_{12}, f_{14}, f_{15}, f_{17}, f_{18}, f_{35} \in M_{20}\left(\Gamma_{0}(12)\right) \backslash S_{20}\left(\Gamma_{0}(12)\right)$ and

$\eta^{a_{1}}(z) \eta^{a_{2}}(2 z) \eta^{a_{3}}(3 z) \eta^{a_{4}}(4 z) \eta^{a_{6}}(6 z) \eta^{a_{12}}(12 z)=\delta\left(b_{1}\right)+\sum_{n=1}^{\infty} c(n) q^{n}$

where for $n \in \mathbb{N}$,

$$
\begin{aligned}
c(n)= & c_{1} \sigma_{19}(n)+c_{2} \sigma_{19}\left(\frac{n}{2}\right)+c_{3} \sigma_{19}\left(\frac{n}{3}\right)+c_{4} \sigma_{19}\left(\frac{n}{4}\right)+c_{6} \sigma_{19}\left(\frac{n}{6}\right)+c_{12} \sigma_{19}\left(\frac{n}{12}\right) \\
& +r_{1} f_{1}(n)+\cdots+r_{35} f_{35}(n) .
\end{aligned}
$$

In particular,

$$
\begin{gathered}
c(2 n)=c_{1} \sigma_{19}(2 n)+c_{2} \sigma_{19}(n)+c_{4} \sigma_{19}\left(\frac{n}{2}\right)+\left(1048577 c_{3}+c_{6}\right) \sigma_{19}\left(\frac{n}{3}\right) \\
+\left(c_{12}-1048576 c_{3}\right) \sigma_{19}\left(\frac{n}{6}\right)+r_{1} f_{1}(2 n)+\cdots+r_{35} f_{35}(2 n), \\
c(2 n-1)=c_{1} \sigma_{19}(2 n-1)+c_{3} \sigma_{19}\left(\frac{2 n-1}{3}\right) \\
+r_{19} f_{19}(2 n-1)+\cdots+r_{35} f_{35}(2 n-1),
\end{gathered}
$$

for $n \in \mathbb{N}$.

Proof: It follows from (6)-(11) that

$a_{1}+2 a_{2}+3 a_{3}+4 a_{4}+6 a_{6}+12 a_{12}=24 b_{1}$,

$a_{1}+a_{2}+a_{3}+a_{4}+a_{6}+a_{12}=40$,

$-\frac{a_{1}}{6}-\frac{a_{2}}{3}-\frac{a_{3}}{6}-\frac{2 a_{4}}{3}-\frac{a_{6}}{3}-\frac{2 a_{12}}{3}=-b_{1}-b_{5}$. 
Now we will use $(p, k)$ parametrization of Alaca, Alaca and Williams, see [1]:

$p(q):=\frac{\varphi^{2}(q)-\varphi^{2}\left(q^{3}\right)}{2 \varphi^{2}\left(q^{3}\right)}, k(q):=\frac{\varphi^{3}\left(q^{3}\right)}{\varphi(q)}$,

where the theta function $\varphi(q)$ is defined by

$\varphi(q)=\sum_{-\infty}^{\infty} q^{n^{2}}$

Setting $x=p$ in (12), and multiplying both sides by $k^{20}$,we obtain

$$
\begin{aligned}
& \frac{k^{20}}{2^{b_{1}+b_{5}}} p^{b_{1}}(1-p)^{b_{2}}(1+p)^{b_{3}}(1+2 p)^{b_{4}}(2+p)^{b_{5}} \\
& =\left(\sum_{i=0}^{40} k_{i} p^{i}\right) k^{20} .
\end{aligned}
$$

Alaca, Alaca and Williams [2] have established the following representations in terms of $p$ and $k$ :

$\eta(q)=2^{-1 / 6} p^{1 / 24}(1-p)^{1 / 2}(1+p)^{1 / 6}(1+2 p)^{1 / 8}(2+p)^{1 / 8} k^{1 / 2}$,

$\eta\left(q^{2}\right)=2^{-1 / 3} p^{1 / 12}(1-p)^{1 / 4}(1+p)^{1 / 12}(1+2 p)^{1 / 4}(2+p)^{1 / 4} k^{1 / 2}$,

$\eta\left(q^{3}\right)=2^{-1 / 6} p^{1 / 8}(1-p)^{1 / 6}(1+p)^{1 / 2}(1+2 p)^{1 / 24}(2+p)^{1 / 24} k^{1 / 2}$,

$\eta\left(q^{4}\right)=2^{-2 / 3} p^{1 / 6}(1-p)^{1 / 8}(1+p)^{1 / 24}(1+2 p)^{1 / 8}(2+p)^{1 / 2} k^{1 / 2}$,

$\eta\left(q^{6}\right)=2^{-1 / 3} p^{1 / 4}(1-p)^{1 / 12}(1+p)^{1 / 4}(1+2 p)^{1 / 12}(2+p)^{1 / 12} k^{1 / 2}$,

$\eta\left(q^{12}\right)=2^{-2 / 3} p^{1 / 2}(1-p)^{1 / 24}(1+p)^{1 / 8}(1+2 p)^{1 / 24}(2+p)^{1 / 6} k^{1 / 2}$,

$$
\begin{aligned}
& E_{6}(q):=1-504 \sum_{n=1}^{\infty} \sigma_{5}(n) q^{n} \\
&=\quad\left(1-246 p-5532 p^{2}-38614 p^{3}-135369 p^{4}-276084 p^{5}\right. \\
&-348024 p^{6}-276084 p^{7}-135369 p^{8}-38614 p^{9}-5532 p^{10} \\
&\left.-246 p^{11}+p^{12}\right) k^{6}, \\
& E_{4}(q):=1+240 \sum_{n=1}^{\infty} \sigma_{3}(n) q^{n} \\
&=\quad\left(1+124 p+964 p^{2}+2788 p^{3}+3910 p^{4}+2788 p^{5}\right. \\
&\left.\quad+964 p^{6}+124 p^{7}+p^{8}\right) k^{4} .
\end{aligned}
$$


Therefore, since

$E_{20}(q)=\frac{121250}{174611} E_{6}^{2}(q) E_{4}^{2}(q)+\frac{53361}{174611} E_{4}^{5}(q)$,

we have

$$
\begin{aligned}
& E_{20}(q)=\left(p^{40}+\frac{3498820}{174611} p^{39}+\frac{1761712380}{174611} p^{38}+\frac{1950771349900}{174611} p^{37}\right. \\
& +\frac{262533149598050}{174611} p^{36}+\frac{12244560329615724}{174611} p^{35} \\
& +\frac{297550209630645660}{174611} p^{34}+\frac{4540576615265413860}{174611} p^{33} \\
& +\frac{48205011029576752215}{174611} p^{32}+\frac{379279886962492426000}{174611} p^{31} \\
& +\frac{2306867772376889866544}{174611} p^{30}+\frac{11172752277687684748080}{174611} p^{29} \\
& +\frac{44032939445831353072280}{174611} p^{28}+\frac{143523588642250460471920}{174611} p^{27} \\
& +\frac{391710935624818929715440}{174611} p^{26}+\frac{903703297519950364759248}{174611} p^{25} \\
& +\frac{1775257667109763890078870}{174611} p^{24}+\frac{2985782926019838802293240}{174611} p^{23} \\
& +\frac{4316791070784960278738760}{174611} p^{22}+\frac{5379734920300102006356840}{174611} p^{21} \\
& +\frac{5788306667816968534061580}{174611} p^{20}+\frac{5379734920300102006356840}{174611} p^{19} \\
& +\frac{4316791070784960278738760}{174611} p^{18}+\frac{2985782926019838802293240}{174611} p^{17} \\
& +\frac{1775257667109763890078870}{174611} p^{16}+\frac{903703297519950364759248}{174611} p^{15} \\
& +\frac{391710935624818929715440}{174611} p^{14}+\frac{143523588642250460471920}{174611} p^{13} \\
& +\frac{44032939445831353072280}{174611} p^{12}+\frac{11172752277687684748080}{174611} p^{11} \\
& +\frac{2306867772376889866544}{174611} p^{10}+\frac{379279886962492426000}{174611} p^{9} \\
& +\frac{48205011029576752215}{174611} p^{8}+\frac{4540576615265413860}{174611} p^{7} \\
& +\frac{297550209630645660}{174611} p^{6}+\frac{12244560329615724}{174611} p^{5}+\frac{262533149598050}{174611} \\
& \left.p^{4}+\frac{1950771349900}{174611} p^{3}+\frac{1761712380}{174611} p^{2}+\frac{3498820}{174611} p+1\right) k^{20},
\end{aligned}
$$




$$
\begin{aligned}
& E_{20}\left(q^{2}\right)=\left(p^{40}+20 p^{39}+\frac{31433280}{174611} p^{38}+\frac{165943150}{174611} p^{37}+\frac{987462125}{174611} p^{36}\right. \\
& +\frac{8929248174}{174611} p^{35}+\frac{303281329710}{174611} p^{34}+\frac{4364341784610}{174611} p^{33} \\
& +\frac{91493235452655}{349222} p^{32}+\frac{361444851257800}{174611} p^{31}+\frac{2201013342701144}{174611} p^{30} \\
& +\frac{10656464415766680}{174611} p^{29}+\frac{41990748446379980}{174611} p^{28} \\
& +\frac{136870946815364920}{174611} p^{27}+\frac{373567300252984440}{174611} p^{26} \\
& +\frac{861845414133190248}{174611} p^{25}+\frac{1693017415776001995}{174611} p^{24} \\
& +\frac{2847457351673446140}{174611} p^{23}+\frac{4116809048699351460}{174611} p^{22} \\
& +\frac{5130518121215151540}{174611} p^{21}+\frac{5520165078218375430}{174611} p^{20} \\
& +\frac{5130518121215151540}{174611} p^{19}+\frac{4116809048699351460}{174611} p^{18} \\
& +\frac{2847457351673446140}{174611} p^{17}+\frac{1693017415776001995}{174611} p^{16} \\
& +\frac{861845414133190248}{174611} p^{15}+\frac{373567300252984440}{174611} p^{14} \\
& +\frac{136870946815364920}{174611} p^{13}+\frac{41990748446379980}{174611} p^{12} \\
& +\frac{10656464415766680}{174611} p^{11}+\frac{2201013342701144}{174611} p^{10} \\
& +\frac{361444851257800}{174611} p^{9}+\frac{91493235452655}{349222} p^{8} \\
& +\frac{4364341784610}{174611} p^{7}+\frac{303281329710}{174611} p^{6}+\frac{8929248174}{174611} p^{5} \\
& \left.+\frac{987462125}{174611} p^{4}+\frac{165943150}{174611} p^{3}+\frac{31433280}{174611} p^{2}+20 p+1\right) k^{20}, \\
& E_{20}\left(q^{3}\right)=\left(p^{40}+20 p^{39}+180 p^{38}+\frac{165882100}{174611} p^{37}+\frac{554420450}{174611} p^{36}\right. \\
& +\frac{1141160724}{174611} p^{35}+\frac{1164216060}{174611} p^{34}+\frac{539536860}{174611} p^{33} \\
& +\frac{8009545815}{174611} p^{32}+\frac{82124642800}{174611} p^{31}+\frac{622305005744}{174611} p^{30} \\
& +\frac{3416310121680}{174611} p^{29}+\frac{13238543613080}{174611} p^{28}+\frac{40264423711120}{174611} p^{27} \\
& +\frac{108624031190640}{174611} p^{26}+\frac{261168091468848}{174611} p^{25}+\frac{521667517163670}{174611} p^{24} \\
& +\frac{854113070472840}{174611} p^{23}+\frac{1212499060327560}{174611} p^{22}+\frac{1543771577414040}{174611} p^{21} \\
& +\frac{1692495554535180}{174611} p^{20}+\frac{1543771577414040}{174611} p^{19} \\
& +\frac{1212499060327560}{174611} p^{18}+\frac{854113070472840}{174611} p^{17}+\frac{521667517163670}{174611} p^{16} \\
& +\frac{261168091468848}{174611} p^{15}+\frac{108624031190640}{174611} p^{14}+\frac{40264423711120}{174611} p^{13} \\
& +\frac{13238543613080}{174611} p^{12}+\frac{3416310121680}{174611} p^{11}+\frac{622305005744}{174611} p^{10} \\
& +\frac{82124642800}{174611} p^{9}+\frac{8009545815}{174611} p^{8}+\frac{539536860}{174611} p^{7}+\frac{1164216060}{174611} p^{6} \\
& \left.+\frac{1141160724}{174611} p^{5}+\frac{554420450}{174611} p^{4}+\frac{165882100}{174611} p^{3}+180 p^{2}+20 p+1\right) k^{20}
\end{aligned}
$$




$$
\begin{aligned}
& E_{20}\left(q^{4}\right)=\left(\frac{1}{1048576} p^{40}+\frac{871405}{45773225984} p^{39}+\frac{440363745}{45773225984} p^{38}\right. \\
& -\frac{117793359275}{11443306496} p^{37}+\frac{23947134429775}{22886612992} p^{36} \\
& -\frac{254868233968161}{11443306496} p^{35}+\frac{302463836011515}{2860826624} p^{34} \\
& +\frac{1160663370518955}{5721653248} p^{33}-\frac{28749261448742445}{22886612992} p^{32} \\
& -\frac{2100550371547975}{1430413312} p^{31}+\frac{8316909253331623}{1430413312} p^{30} \\
& +\frac{2649051448593615}{357603328} p^{29}-\frac{9448705577013595}{715206656} p^{28} \\
& -\frac{3737818023814145}{178801664} p^{27}+\frac{195006967788285}{11175104} p^{26} \\
& +\frac{3816495781888401}{89400832} p^{25}+\frac{3875892669469395}{715206656} p^{24} \\
& -\frac{4402258116918165}{178801664} p^{23}+\frac{1872902744061915}{178801664} p^{22} \\
& +\frac{2346702805920615}{44700416} p^{21}+\frac{4147844506625385}{89400832} p^{20} \\
& +\frac{951687682156815}{44700416} p^{19}+\frac{147096539206365}{11175104} p^{18} \\
& +\frac{331640077115295}{22350208} p^{17}+\frac{1052959610032065}{89400832} p^{16} \\
& +\frac{62680520061747}{11175104} p^{15}+\frac{19650005782785}{11175104} p^{14} \\
& +\frac{1564837152745}{2793776} p^{13}+\frac{1661596819735}{5587552} p^{12} \\
& +\frac{24368406705}{174611} p^{11}+\frac{3494456413}{349222} p^{10}-\frac{7531426175}{174611} p^{9} \\
& -\frac{93384029385}{2793776} p^{8}-\frac{5207303235}{698444} p^{7}+\frac{4219951665}{698444} p^{6} \\
& \left.+\frac{1140923124}{174611} p^{5}+\frac{554390750}{174611} p^{4}+950 p^{3}+180 p^{2}+20 p+1\right) k^{20} \text {, }
\end{aligned}
$$$$
E_{20}\left(q^{6}\right)=\left(p^{40}+20 p^{39}+180 p^{38}+950 p^{37}+3175 p^{36}+6534 p^{35}\right.
$$$$
+\frac{1054868910}{174611} p^{34}-\frac{1302376290}{174611} p^{33}-\frac{11730204945}{349222} p^{32}
$$$$
-\frac{7966512200}{174611} p^{31}-\frac{1308580456}{174611} p^{30}+\frac{12073490280}{174611} p^{29}
$$$$
+\frac{18399531980}{174611} p^{28}+\frac{6936773320}{174611} p^{27}-\frac{12990377160}{174611} p^{26}
$$$$
-\frac{20113138152}{174611} p^{25}-\frac{7670974005}{174611} p^{24}+\frac{8584483140}{174611} p^{23}
$$$$
+\frac{11628731460}{174611} p^{22}+\frac{3054606540}{174611} p^{21}-\frac{2512795770}{174611} p^{20}
$$$$
+\frac{3054606540}{174611} p^{19}+\frac{11628731460}{174611} p^{18}+\frac{8584483140}{174611} p^{17}
$$$$
-\frac{7670974005}{174611} p^{16}-\frac{20113138152}{174611} p^{15}-\frac{12990377160}{174611} p^{14}
$$$$
+\frac{6936773320}{174611} p^{13}+\frac{18399531980}{174611} p^{12}+\frac{12073490280}{174611} p^{11}
$$$$
-\frac{1308580456}{174611} p^{10}-\frac{7966512200}{174611} p^{9}-\frac{11730204945}{349222} p^{8}
$$$$
-\frac{1302376290}{174611} p^{7}+\frac{1054868910}{174611} p^{6}+6534 p^{5}+3175 p^{4}+950 p^{3}
$$$$
\left.+180 p^{2}+20 p+1\right) k^{20},
$$ 


$$
\begin{aligned}
E_{20}\left(q^{12}\right)= & \left(\frac{1}{1048576} p^{40}+\frac{5}{262144} p^{39}+\frac{45}{262144} p^{38}+\frac{10367425}{11443306496} p^{37}\right. \\
& +\frac{69294925}{22886612992} p^{36}+\frac{71290989}{11443306496} p^{35}+\frac{36305955}{5721653248} p^{34} \\
& +\frac{16633755}{5721653248} p^{33}+\frac{999590355}{22886612992} p^{32}+\frac{173342075}{1430413312} p^{31} \\
& -\frac{2395113227}{1430413312} p^{30}-\frac{5705331435}{357603328} p^{29}-\frac{44179109845}{715206656} p^{28} \\
& -\frac{16087374695}{178801664} p^{27}+\frac{19855618155}{89400832} p^{26}+\frac{124711192701}{89400832} p^{25} \\
& +\frac{1987639824195}{715206656} p^{24}+\frac{99698108985}{178801664} p^{23}-\frac{1697890834635}{178801664} p^{22} \\
& -\frac{935935221585}{44700416} p^{21}-\frac{1056483866565}{89400832} p^{20}+\frac{1341018629565}{44700416} p^{19} \\
& +\frac{1552706681055}{22350208} p^{18}+\frac{982091824695}{22350208} p^{17}-\frac{4430721627135}{89400832} p^{16} \\
& -\frac{1318772631303}{11175104} p^{15}-\frac{840497560965}{11175104} p^{14}+\frac{110625563695}{2793776} p^{13} \\
& +\frac{588727287385}{5587552} p^{12}+\frac{138285}{2} p^{11}-7496 p^{10}-45625 p^{9}-\frac{537435}{16} p^{8} \\
& \left.-\frac{29835}{4} p^{7}+\frac{24165}{4} p^{6}+6534 p^{5}+3175 p^{4}+950 p^{3}+180 p^{2}+20 p+1\right) k^{20} .
\end{aligned}
$$

We can also similarly determine $f_{1}, \cdots, f_{30}$ and $f_{31}$ in terms of $p$ and $k$ as in www.bariskendirli.com.tr/weight20/Table 2. Obviously, $f_{1}, \ldots f_{35}$ are functions of $q$, see (3),(15). We see that $\left\{f_{1}, \ldots f_{35}\right\} \backslash\left\{f_{7}, f_{12}, f_{14}, f_{15}, f_{17}, f_{18}, f_{35}\right\} \in$ $S_{20}\left(\Gamma_{0}(12)\right), f_{7}, f_{12}, f_{14}, f_{15}, f_{17}, f_{18}, f_{35} \in M_{20}\left(\Gamma_{0}(12)\right) \backslash S_{20}\left(\Gamma_{0}(12)\right)$ by [4]. Now

$$
\begin{aligned}
& \eta^{a_{1}}(z) \eta^{a_{2}}(2 z) \eta^{a_{3}}(3 z) \eta^{a_{4}}(4 z) \eta^{a_{6}}(6 z) \eta^{a_{12}}(12 z) \\
& =q^{b_{1}} \prod_{n=1}^{\infty}\left(1-q^{n}\right)^{a_{1}}\left(1-q^{2 n}\right)^{a_{2}}\left(1-q^{3 n}\right)^{a_{3}}\left(1-q^{4 n}\right)^{a_{4}}\left(1-q^{6 n}\right)^{a_{6}}\left(1-q^{12 n}\right)^{a_{12}} \\
& =2^{-\frac{a_{1}}{6}-\frac{a_{2}}{3}-\frac{a_{3}}{6}-\frac{2 a_{4}}{3}-\frac{a_{6}}{3}-\frac{2 a_{12}}{3}} p^{\frac{a_{1}}{24}+\frac{a_{2}}{12}+\frac{a_{3}}{8}+\frac{a_{4}}{6}+\frac{a_{6}}{4}+\frac{a_{12}}{2}}(1-p)^{\frac{a_{1}}{2}+\frac{a_{2}}{4}+\frac{a_{3}}{6}+\frac{a_{4}}{8}+\frac{a_{6}}{12}+\frac{a_{12}}{24}} \\
& (1+p)^{\frac{a_{1}}{6}+\frac{a_{2}}{12}+\frac{a_{3}}{2}+\frac{a_{4}}{24}+\frac{a_{6}}{4}+\frac{a_{12}}{8}}(1+2 p)^{\frac{a_{1}}{8}+\frac{a_{2}}{4}+\frac{a_{3}}{24}+\frac{a_{4}}{8}+\frac{a_{6}}{12}+\frac{a_{12}}{24}}(2+p)^{\frac{a_{1}}{8}+\frac{a_{2}}{4}+\frac{a_{3}}{24}+\frac{a_{4}}{2}+\frac{a_{6}}{12}+\frac{a_{12}}{6}} \\
& k^{\frac{a_{1}+a_{2}+a_{3}+a_{4}+a_{6}+a_{12}}{2}}=\frac{k^{20}}{2^{b_{1}+b_{5}}} p^{b_{1}}(1-p)^{b_{2}}(1+p)^{b_{3}}(1+2 p)^{b_{4}}(2+p)^{b_{5}}
\end{aligned}
$$$$
=\left(\sum_{i=0}^{40} k_{i} p^{i}\right) k^{20}
$$ 


$$
\begin{aligned}
& =\frac{174611 c_{1}}{13200}\left(1+\frac{13200}{174611} \sum_{n=1}^{\infty} \sigma_{19}(n) q^{n}\right)+\frac{174611 c_{2}}{13200}\left(1+\frac{13200}{174611} \sum_{n=1}^{\infty} \sigma_{19}(n) q^{2 n}\right) \\
& +\frac{174611 c_{3}}{13200}\left(1+\frac{13200}{174611} \sum_{n=1}^{\infty} \sigma_{19}(n) q^{3 n}\right)+\frac{174611 c_{4}}{13200}\left(1+\frac{13200}{174611} \sum_{n=1}^{\infty} \sigma_{19}(n) q^{4 n}\right) \\
& +\frac{174611 c_{6}}{13200}\left(1+\frac{13200}{174611} \sum_{n=1}^{\infty} \sigma_{19}(n) q^{6 n}\right)+\frac{174611 c_{12}}{13200}\left(1+\frac{13200}{174611} \sum_{n=1}^{\infty} \sigma_{19}(n) q^{12 n}\right) \\
& +r_{1} q^{7} \prod_{n=1}^{\infty} \frac{\left(1-q^{4 n}\right)^{43}\left(1-q^{6 n}\right)^{43}}{\left(1-q^{2 n}\right)^{29}\left(1-q^{12 n}\right)^{17}} \\
& +r_{2} q^{7} \prod_{n=1}^{\infty} \frac{\left(1-q^{4 n}\right)^{38}\left(1-q^{6 n}\right)^{48}}{\left(1-q^{2 n}\right)^{28}\left(1-q^{12}\right)^{18}} \\
& +r_{3} q^{9} \prod_{n=1}^{\infty} \frac{\left(1-q^{4 n}\right)^{50}\left(1-q^{6 n}\right)^{24}}{\left(1-q^{2 n}\right)^{28}\left(1-q^{12 n}\right)^{6}} \\
& +r_{4} q^{3} \prod_{n=1}^{\infty} \frac{\left(1-q^{4 n}\right)^{50}\left(1-q^{6 n}\right)^{48}}{\left(1-q^{2 n}\right)^{28}\left(1-q^{12 n}\right)^{30}} \\
& +r_{5} \cdot q^{9} \prod_{n=1}^{\infty} \frac{\left(\left(1-q^{4 n}\right)^{45}\left(1-q^{6 n}\right)^{29}\right.}{\left(1-q^{2 n}\right)^{27}\left(1-q^{12 n}\right)^{7}} \\
& +r_{6} q^{9} \prod_{n=1}^{\infty} \frac{\left(1-q^{4 n}\right)^{40}\left(1-q^{6 n}\right)^{34}}{\left(1-q^{2 n}\right)^{26}\left(1-q^{12 n}\right)^{8}} \\
& +r_{7} q^{19} \prod_{n=1}^{\infty} \frac{\left(1-q^{4 n}\right)^{44}\left(1-q^{12 n}\right)^{36}}{\left(1-q^{2 n}\right)^{22}\left(1-q^{6 n}\right)^{18}} \\
& +r_{8} q^{19} \prod_{n=1}^{\infty} \frac{\left(1-q^{4 n}\right)^{47}\left(1-q^{6 n}\right)^{15}\left(1-q^{12 n}\right)^{3}}{\left(1-q^{2 n}\right)^{25}} \\
& +r_{9} q^{5} \prod_{n=1}^{\infty} \frac{\left(1-q^{4 n}\right)^{47}\left(1-q^{6 n}\right)^{39}}{\left(1-q^{2 n}\right)^{25}\left(1-q^{12 n}\right)^{21}} \\
& +r_{10} q^{9} \prod_{n=1}^{\infty} \frac{\left(1-q^{4 n}\right)^{30}\left(1-q^{6 n}\right)^{44}}{\left(1-q^{2 n}\right)^{24}\left(1-q^{12 n}\right)^{10}}
\end{aligned}
$$




$$
\begin{aligned}
& +r_{11} q^{13} \prod_{n=1}^{\infty} \frac{\left(1-q^{4 n}\right)^{44}\left(1-q^{6 n}\right)^{6}\left(1-q^{12 n}\right)^{12}}{\left(1-q^{2 n}\right)^{22}} \\
& +r_{12} q^{15} \prod_{n=1}^{\infty} \frac{\left(1-q^{2 n}\right)^{15}\left(1-q^{4 n}\right)^{35}\left(1-q^{12 n}\right)^{11}}{\left(1-q^{6 n}\right)^{21}} \\
& +r_{13} q^{9} \prod_{n=1}^{\infty} \frac{\left(1-q^{4 n}\right)^{25}\left(1-q^{6 n}\right)^{49}}{\left(1-q^{2 n}\right)^{23}\left(1-q^{12 n}\right)^{11}} \\
& +r_{14} q^{17} \prod_{n=1}^{\infty} \frac{\left(1-q^{4 n}\right)^{27}\left(1-q^{6 n}\right)^{11}\left(1-q^{12 n}\right)^{23}}{\left(1-q^{2 n}\right)^{21}} \\
& +r_{15} q^{15} \prod_{n=1}^{\infty} \frac{\left(1-q^{4 n}\right)^{5}\left(1-q^{6 n}\right)^{45}\left(1-q^{12 n}\right)^{9}}{\left(1-q^{2 n}\right)^{19}} \\
& +r_{16} q^{13} \prod_{n=1}^{\infty} \frac{\left(1-q^{4 n}\right)^{49}\left(1-q^{6 n}\right)^{23}\left(1-q^{12 n}\right)^{13}}{\left(1-q^{2 n}\right)^{23}} \\
& +r_{17} q^{17} \prod_{n=1}^{\infty} \frac{\left(1-q^{4 n}\right)^{43}\left(1-q^{12 n}\right)^{31}}{\left(1-q^{2 n}\right)^{17}\left(1-q^{6 n}\right)^{17}} \\
& +r_{18} q^{15} \prod_{n=1}^{\infty} \frac{\left(1-q^{6 n}\right)^{50}\left(1-q^{12 n}\right)^{8}}{\left(1-q^{2 n}\right)^{18}} \prod_{n=1}^{\infty} \\
& +r_{22} q^{12} \prod_{n=1}^{\infty} \frac{\left(1-q^{2 n}\right)\left(1-q^{4 n}\right)^{19}\left(1-q^{6 n}\right)^{13}\left(1-q^{4 n}\right)^{15}\left(1-q^{6 n}\right)^{17}\left(1-q^{12 n}\right)^{11}}{\left(1-q^{2 n}\right)^{3}} \\
& +r_{19} q^{16} \prod_{n=1}^{\infty} \frac{\left(1-q^{4 n}\right)^{12}\left(1-q^{6 n}\right)^{20}\left(1-q^{12 n}\right)^{20}}{\left(1-q^{2 n}\right)^{12}} \\
& +r_{n=1}^{\infty} \frac{\left(1-q^{2 n}\right)^{11}\left(1-q^{4 n}\right)^{17}\left(1-q^{6 n}\right)^{15}}{\left(1-q^{12 n}\right)^{3}} \\
& +r_{21} q^{14} \prod_{n=1}^{\infty} \frac{\left(1-q^{4 n}\right)^{16}\left(1-q^{6 n}\right)^{16}\left(1-q^{12 n}\right)^{16}}{\left(1-q^{4 n}\right)^{20}\left(1-q^{6 n}\right)^{12}\left(1-q^{12 n}\right)^{12}}
\end{aligned}
$$




$$
\begin{aligned}
& +r_{26} q^{14} \prod_{n=1}^{\infty}\left(1-q^{2 n}\right)^{2}\left(1-q^{4 n}\right)^{2}\left(1-q^{6 n}\right)^{18}\left(1-q^{12 n}\right)^{18} \\
& +r_{27} q^{14} \prod_{n=1}^{\infty} \frac{\left(1-q^{2 n}\right)^{12}\left(1-q^{6 n}\right)^{20}\left(1-q^{12 n}\right)^{20}}{\left(1-q^{4 n}\right)^{12}} \\
& +r_{28} q^{12} \prod_{n=1}^{\infty}\left(1-q^{2 n}\right)^{4}\left(1-q^{4 n}\right)^{16}\left(1-q^{6 n}\right)^{4}\left(1-q^{12 n}\right)^{16} \\
& +r_{29} q^{12} \prod_{n=1}^{\infty} \frac{\left(1-q^{2 n}\right)^{16}\left(1-q^{6 n}\right)^{16}\left(1-q^{12 n}\right)^{16}}{\left(1-q^{4 n}\right)^{8}} \\
& +r_{30} q^{10} \prod_{n=1}^{\infty} \frac{\left(1-q^{2 n}\right)^{16}\left(1-q^{4 n}\right)^{16}\left(1-q^{12 n}\right)^{16}}{\left(1-q^{6 n}\right)^{8}} \\
& +r_{31} q^{8} \prod_{n=1}^{\infty}\left(1-q^{2 n}\right)^{6}\left(1-q^{4 n}\right)^{18}\left(1-q^{6 n}\right)^{14}\left(1-q^{12 n}\right)^{2} \\
& +r_{32} q^{4} \prod_{n=1}^{\infty} \frac{\left(1-q^{2 n}\right)^{16}\left(1-q^{4 n}\right)^{16}\left(1-q^{6 n}\right)^{16}}{\left(1-q^{12 n}\right)^{8}} \\
& +r_{33} q^{8} \prod_{n=1}^{\infty} \frac{\left(1-q^{2 n}\right)^{20}\left(1-q^{4 n}\right)^{20}\left(1-q^{12 n}\right)^{12}}{\left(1-q^{6 n}\right)^{12}} \\
& +r_{34} q^{2} \prod_{n=1}^{\infty} \frac{\left(1-q^{2 n}\right)^{20}\left(1-q^{4 n}\right)^{20}\left(1-q^{6 n}\right)^{12}}{\left(1-q^{12 n}\right)^{12}} \\
& +r_{35} \prod_{n=1}^{\infty} \frac{\left(1-q^{2 n}\right)^{30}\left(1-q^{4 n}\right)^{30}}{\left(1-q^{6 n}\right)^{10}\left(1-q^{12 n}\right)^{10}} \\
& =\delta\left(b_{1}\right)+\sum_{n=1}^{\infty}\left(c_{1} \sigma_{19}(n)+c_{2} \sigma_{19}\left(\frac{n}{2}\right)+c_{3} \sigma_{19}\left(\frac{n}{3}\right)+c_{4} \sigma_{19}\left(\frac{n}{4}\right)\right. \\
& \left.+c_{6} \sigma_{19}\left(\frac{n}{6}\right)+c_{12} \sigma_{19}\left(\frac{n}{12}\right)\right)+r_{1} f_{1}(n)+\ldots .+r_{35} f_{35}(n),
\end{aligned}
$$

where

$$
\delta\left(b_{1}\right)=\left\{\begin{array}{l}
0 \text { if } b_{1} \neq 0 \\
1 \text { if } b_{1}=0
\end{array} .\right.
$$

So

$$
\begin{aligned}
c(n)= & \left(c_{1} \sigma_{19}(n)+c_{2} \sigma_{19}\left(\frac{n}{2}\right)+c_{3} \sigma_{19}\left(\frac{n}{3}\right)+c_{4} \sigma_{19}\left(\frac{n}{4}\right)\right. \\
& \left.+c_{6} \sigma_{19}\left(\frac{n}{6}\right)+c_{12} \sigma_{19}\left(\frac{n}{12}\right)\right)+r_{1} f_{1}(n)+\ldots .+r_{35} f_{35}(n) .
\end{aligned}
$$

Therefore, for $\mathrm{n}=1,2, \ldots$,

$$
\begin{gathered}
c(2 n)=c_{1} \sigma_{19}(2 n)+c_{2} \sigma_{19}(n)+c_{4} \sigma_{19}\left(\frac{n}{2}\right)+\left(1048577 c_{3}+c_{6}\right) \sigma_{19}\left(\frac{n}{3}\right) \\
+\left(c_{12}-1048576 c_{3}\right) \sigma_{19}\left(\frac{n}{6}\right)+r_{19} f_{19}(2 n)+\cdots+r_{35} f_{35}(2 n), \\
c(2 n-1)=c_{1} \sigma_{19}(2 n-1)+c_{3} \sigma_{19}\left(\frac{2 n-1}{3}\right) \\
\quad+r_{1} f_{1}(2 n-1)+\cdots+r_{18} f_{18}(2 n-1),
\end{gathered}
$$


since it is easy to see that

$\sigma_{k}\left(\frac{2 n}{3}\right)=\left(2^{k}+1\right) \sigma_{k}\left(\frac{n}{3}\right)-2^{k} \sigma_{k}\left(\frac{n}{6}\right)$

hence,

$\sigma_{19}\left(\frac{2 n}{3}\right)=1048577 \sigma_{19}\left(\frac{n}{3}\right)-1048576 \sigma_{19}\left(\frac{n}{6}\right)$

and use the Lemma before the Theorem.

\section{Conclusion}

We have found 570 eta quotients, see Table 4 , such that, for $n=1,2, \cdots$,

$$
\begin{aligned}
& c(2 n)=c_{1} \sigma_{19}(2 n)+c_{2} \sigma_{19}(n)+c_{4} \sigma_{19}\left(\frac{n}{2}\right)+\left(1048577 c_{3}+c_{6}\right) \sigma_{19}\left(\frac{n}{3}\right) \\
& +\left(c_{12}-1048576 c_{3}\right) \sigma_{19}\left(\frac{n}{6}\right) \\
c(2 n-1)= & c_{1} \sigma_{19}(2 n-1)+c_{3} \sigma_{19}\left(\frac{2 n-1}{3}\right)+r_{1} f_{1}(2 n-1)+\cdots+r_{18} f_{18}(2 n-1) .
\end{aligned}
$$

and 1208 eta quotients, such that for $n=1,2, \cdots$,

$$
\begin{aligned}
c(2 n)= & c_{1} \sigma_{19}(2 n)+c_{2} \sigma_{19}(n)+c_{4} \sigma_{19}\left(\frac{n}{2}\right)+c_{6} \sigma_{19}\left(\frac{n}{3}\right) \\
& +c_{12} \sigma_{19}\left(\frac{n}{6}\right)+r_{19} f_{19}(2 n)+\cdots+r_{35} f_{35}(2 n), \\
c(2 n-1)= & 0 .
\end{aligned}
$$

Moreover, if $f$ is an eta quotient, then the coefficients of $\frac{1}{2}(f(q)+f(-q))$ are exactly the even coefficients of $f$. In particular, it means that we have obtained all coefficients of some sum of 570 eta quotients. 


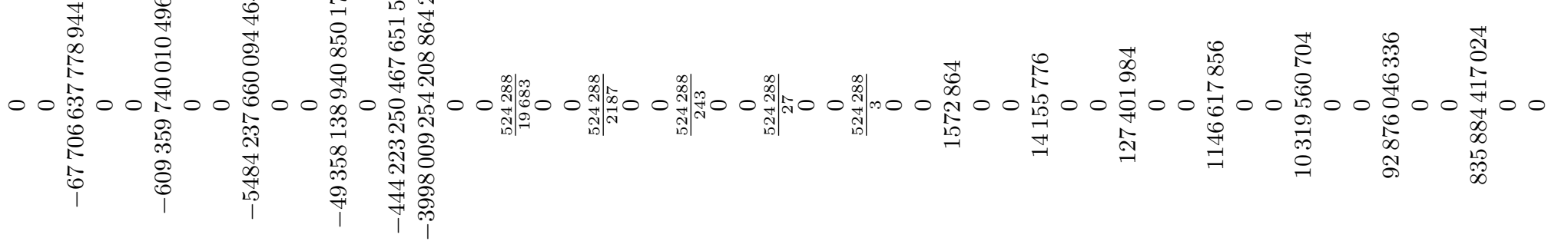

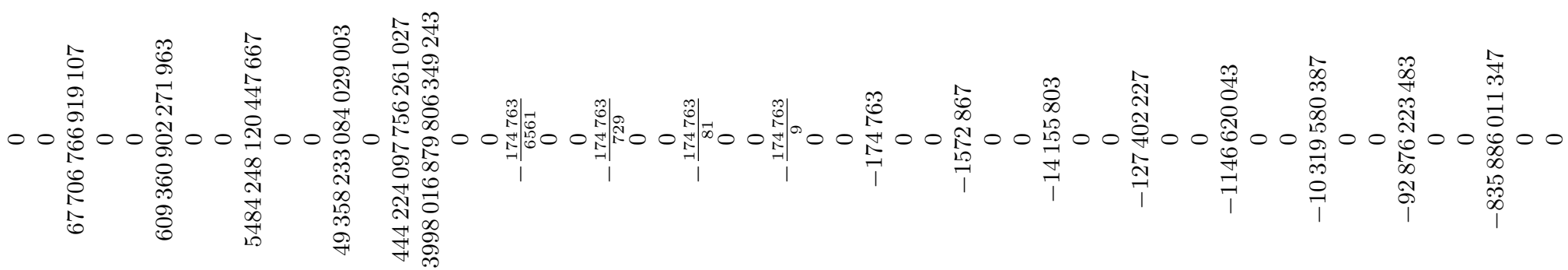

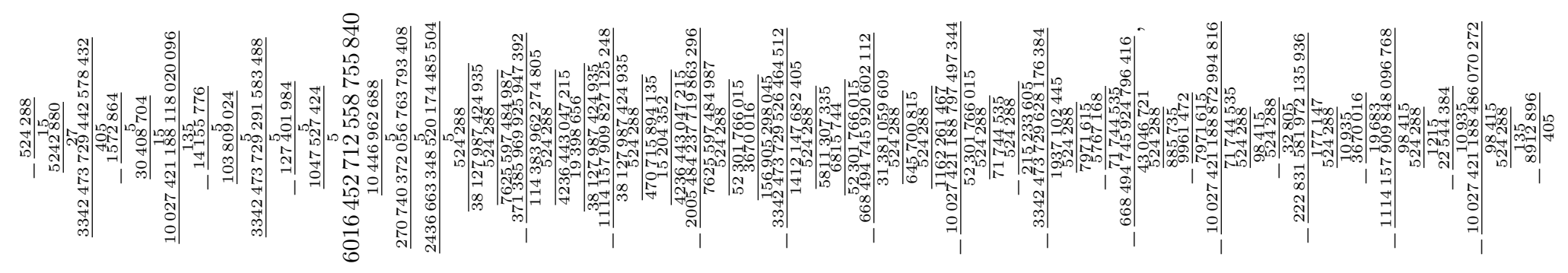

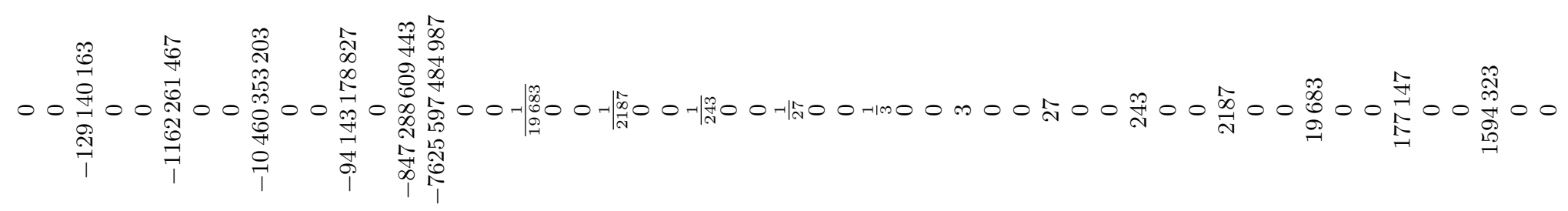

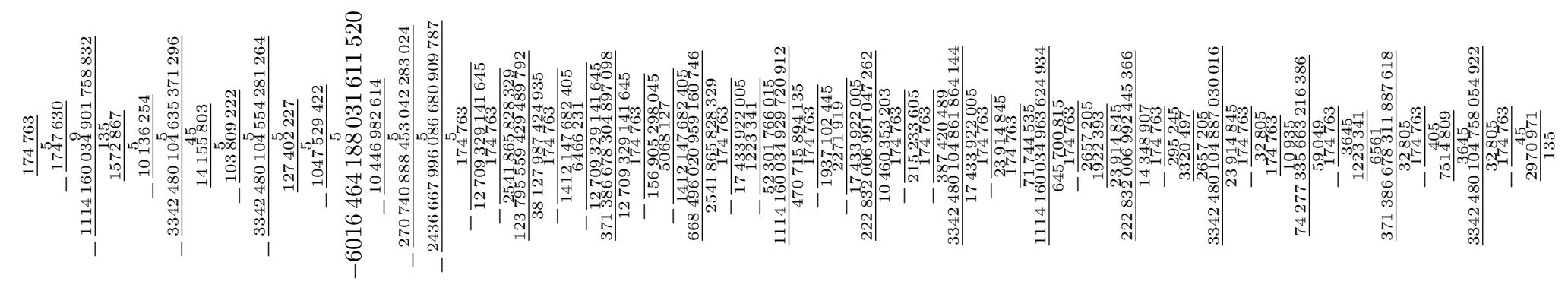

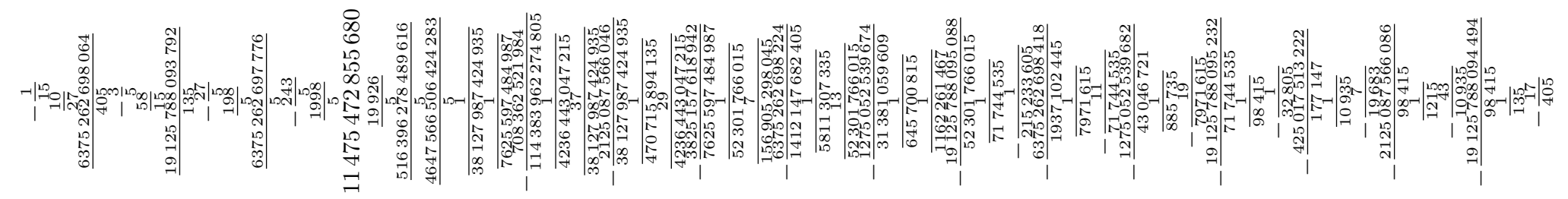

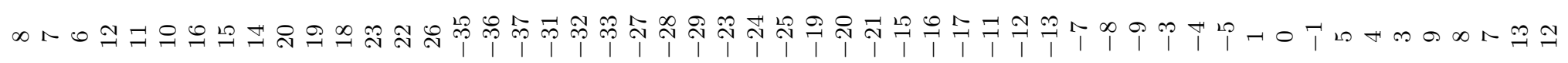

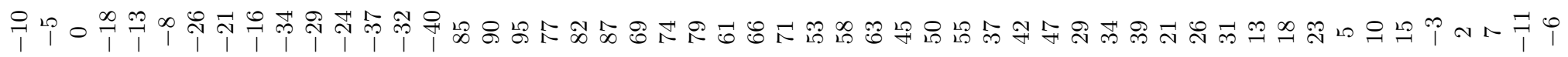

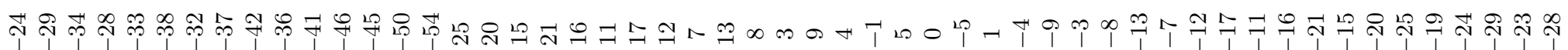

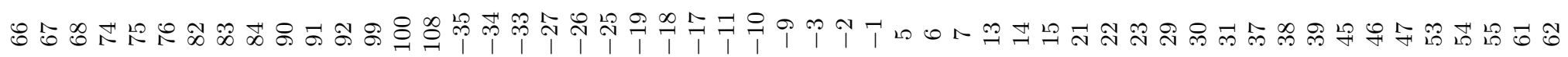
동 


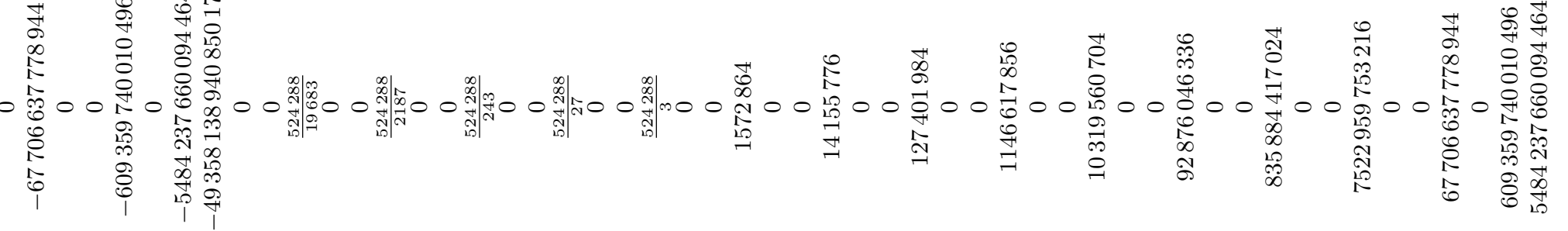

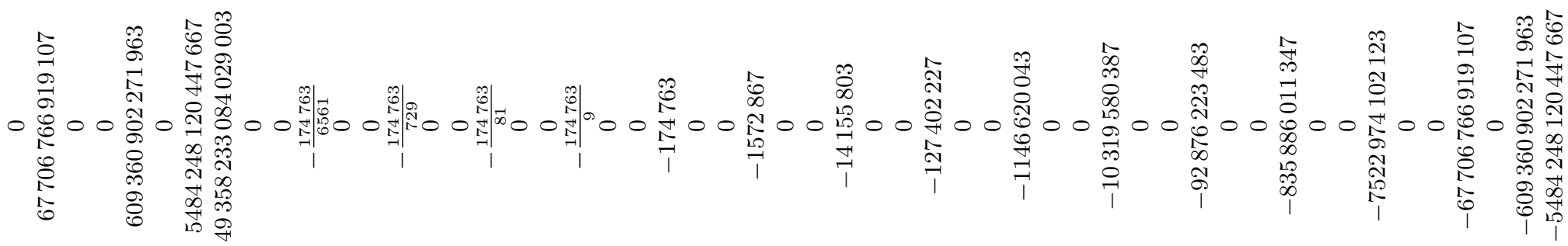

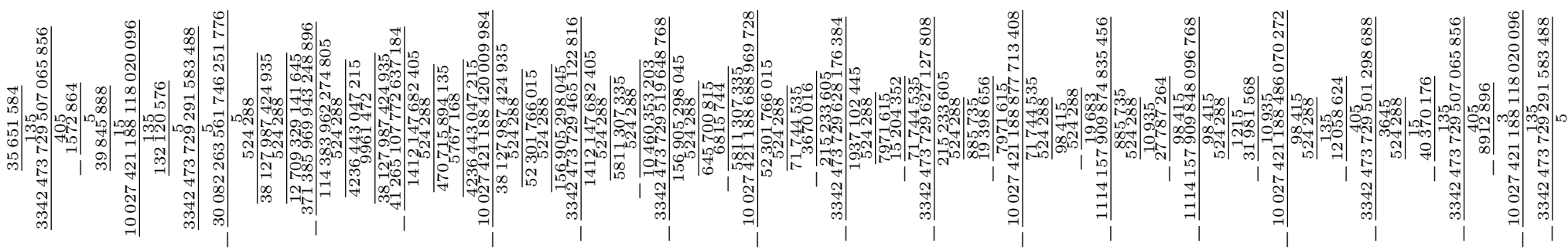

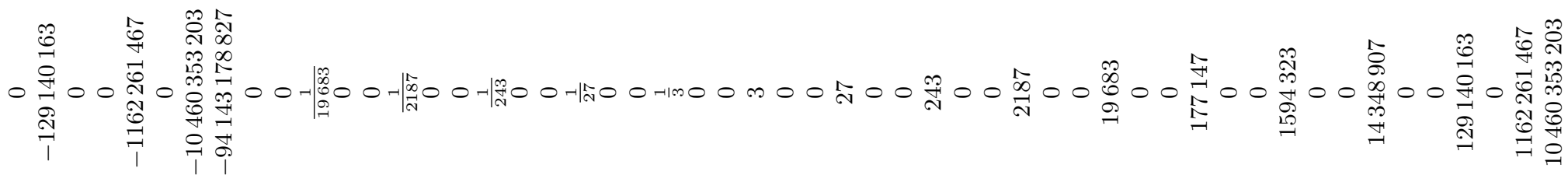
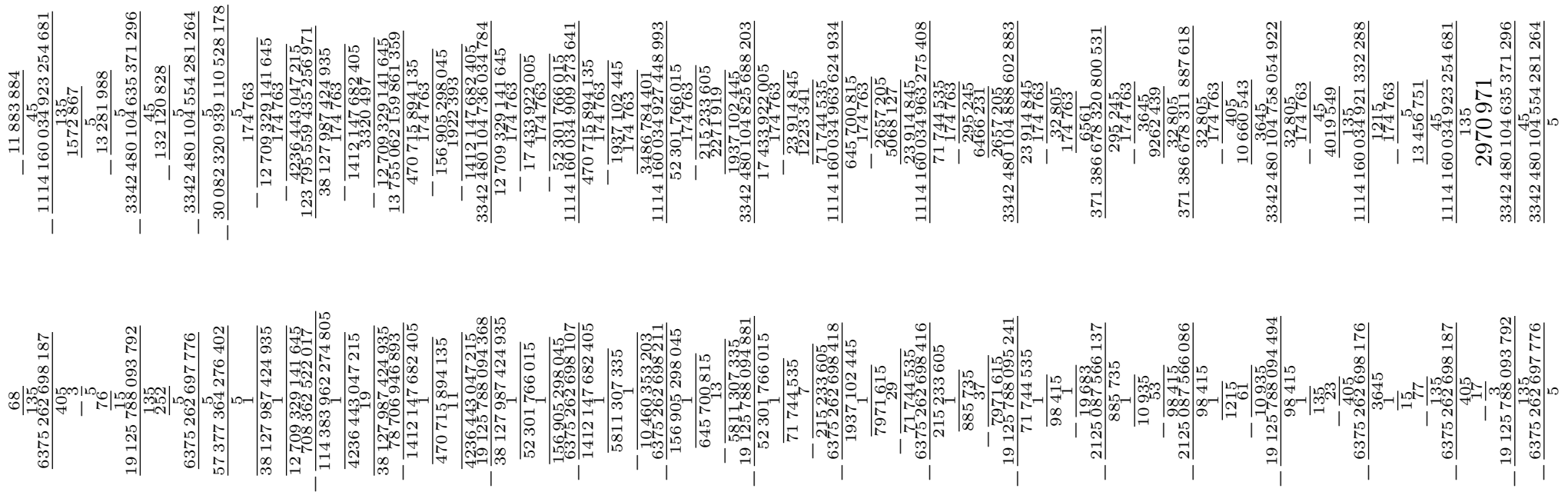

哭

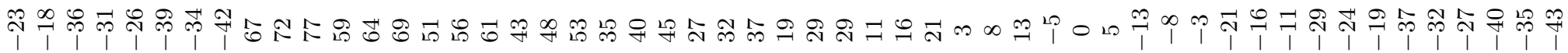
땀유 ア

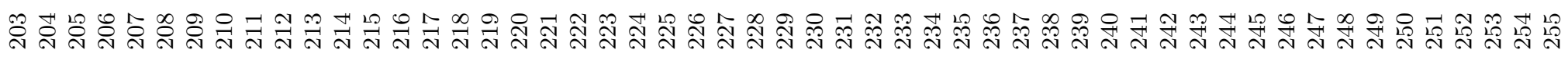




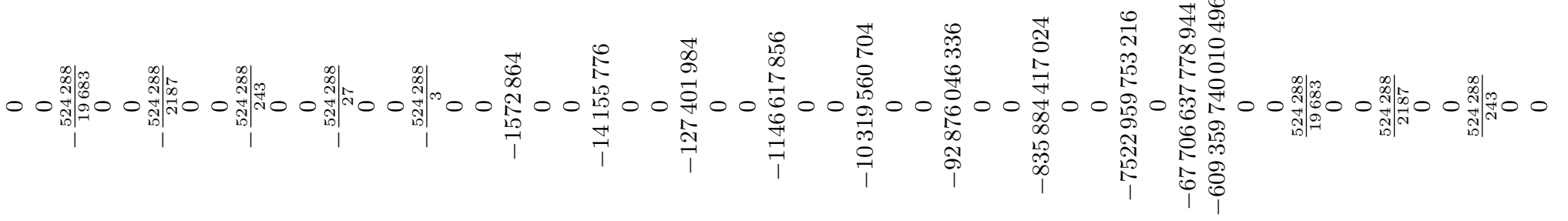

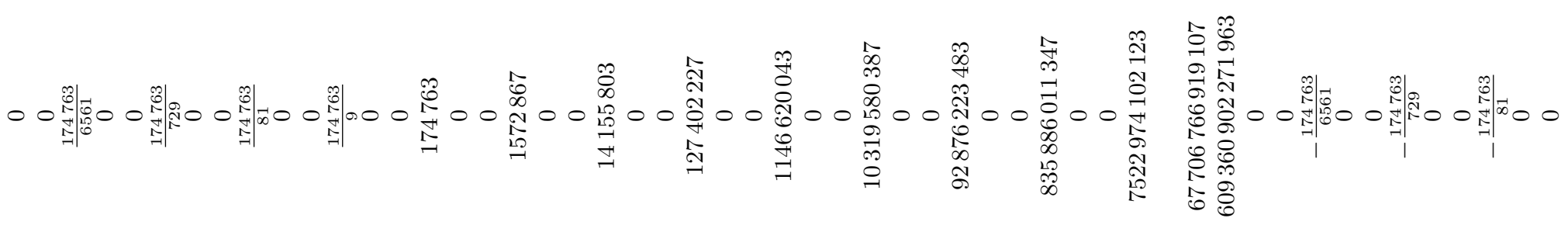

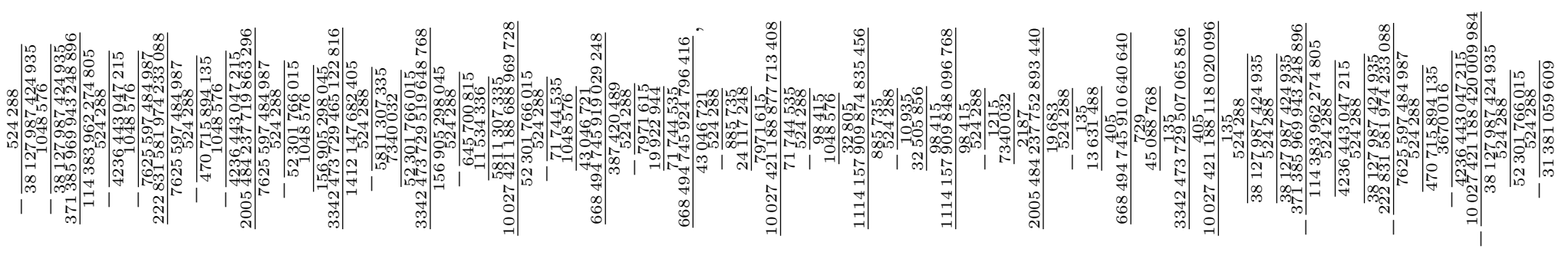

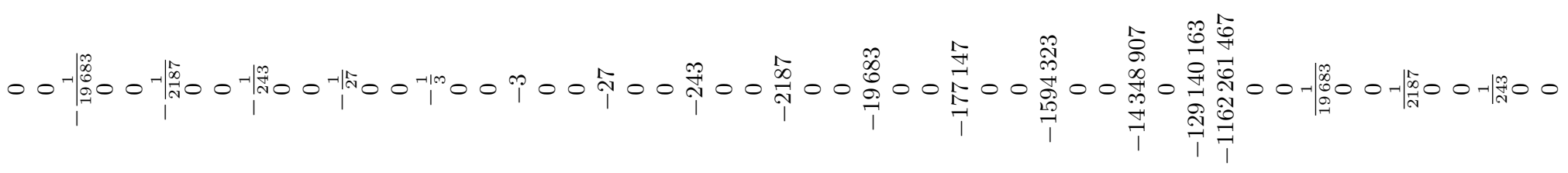

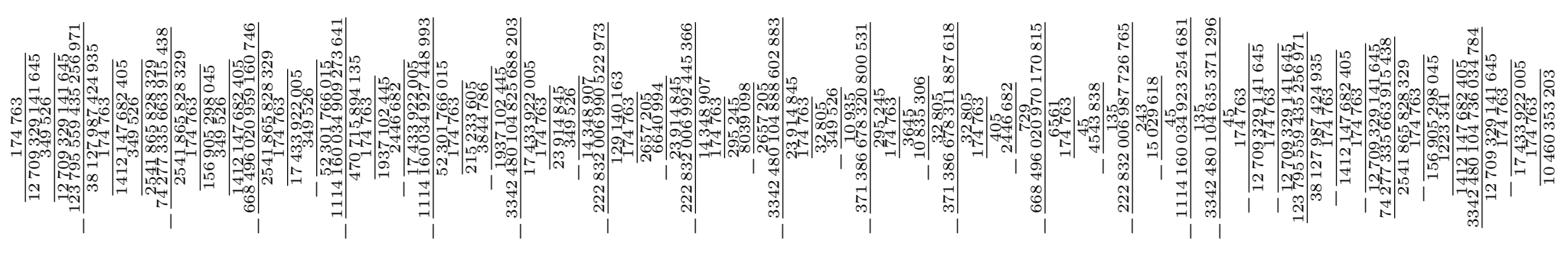

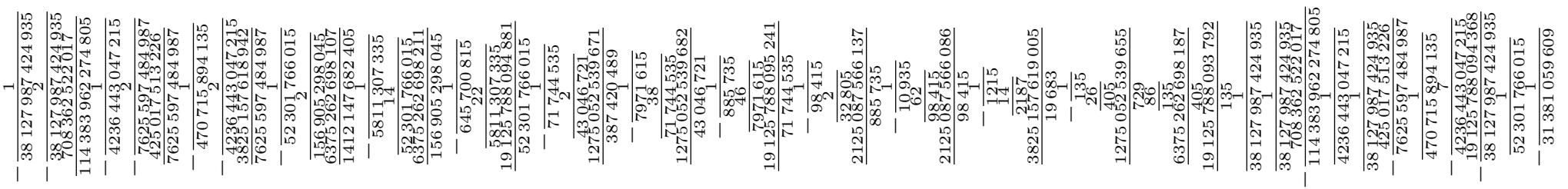

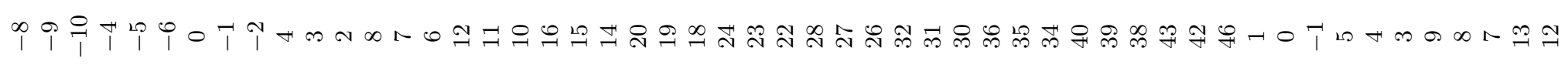

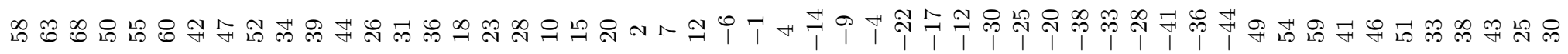

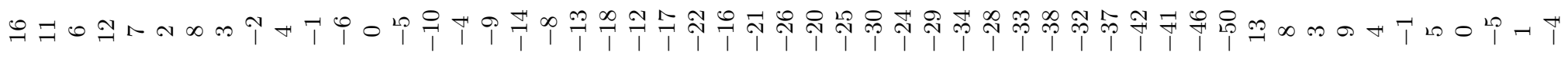

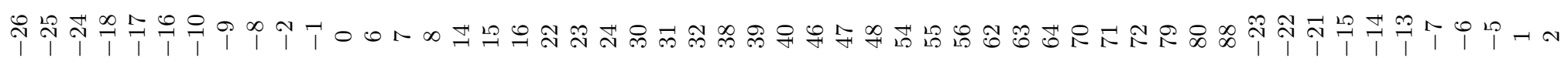

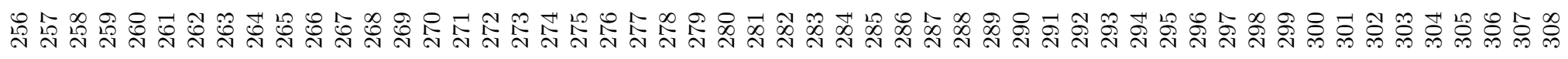




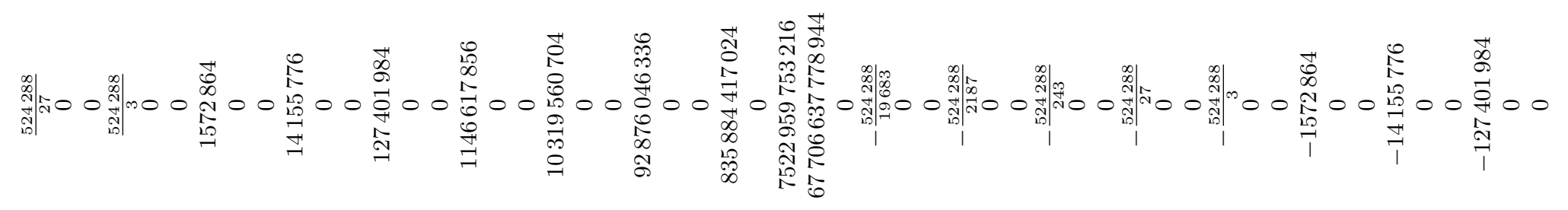

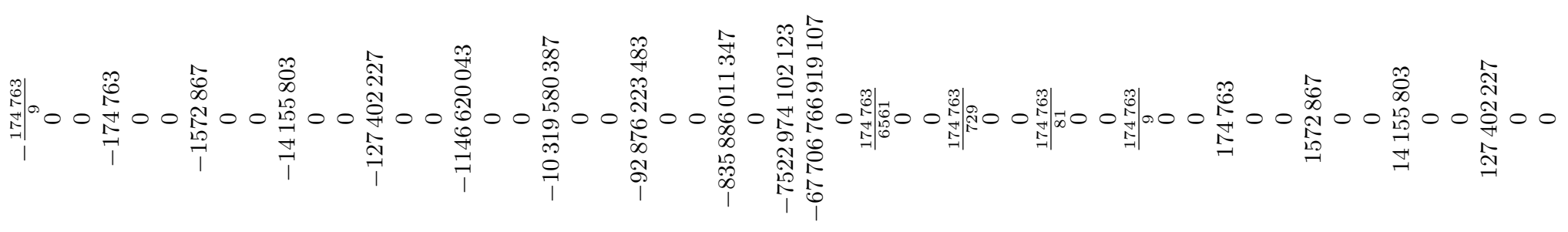

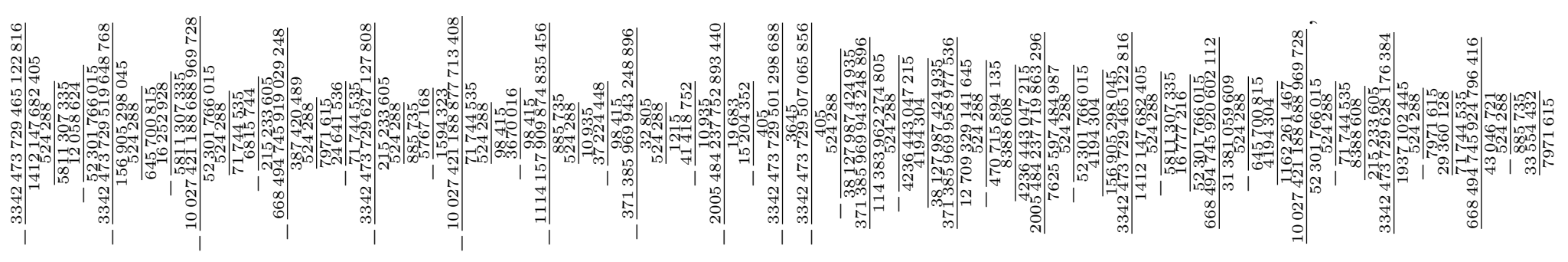

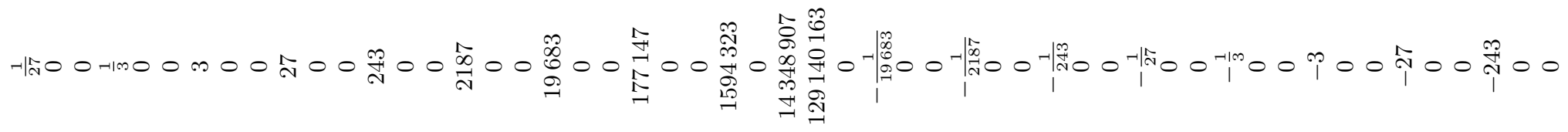
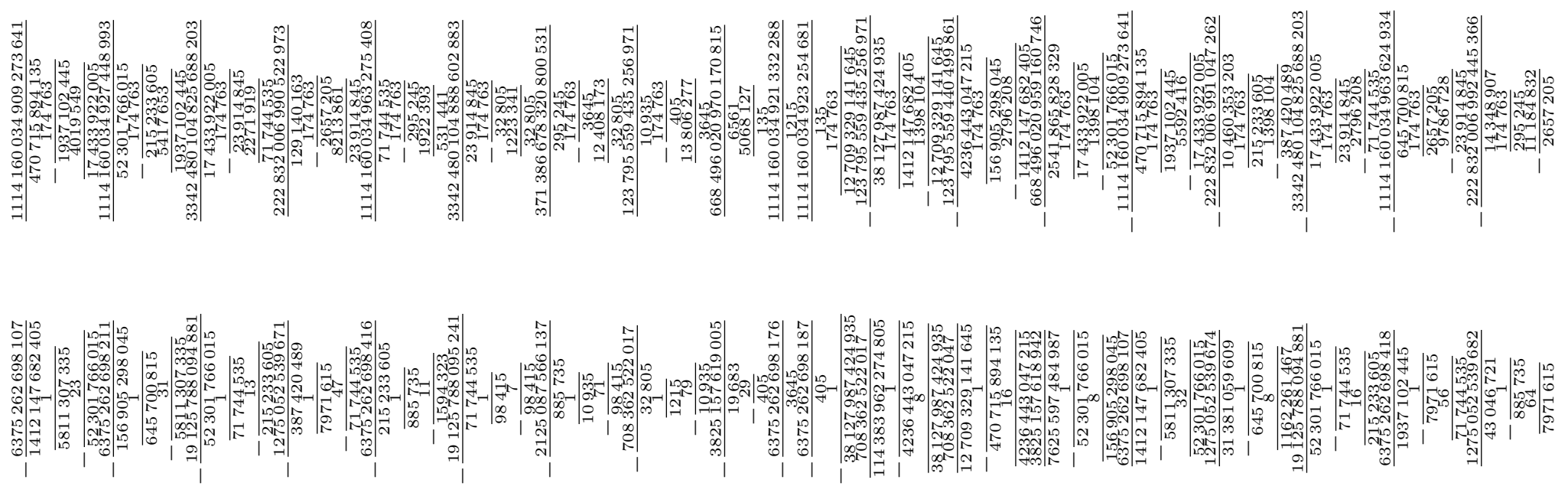

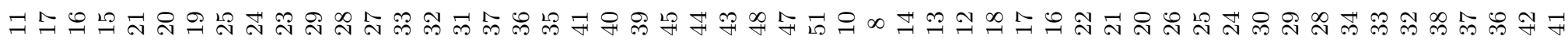
象좃 ஒ

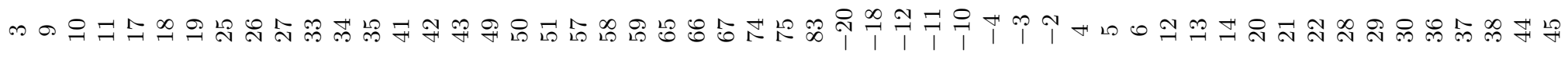

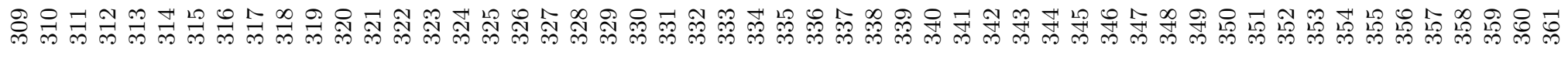




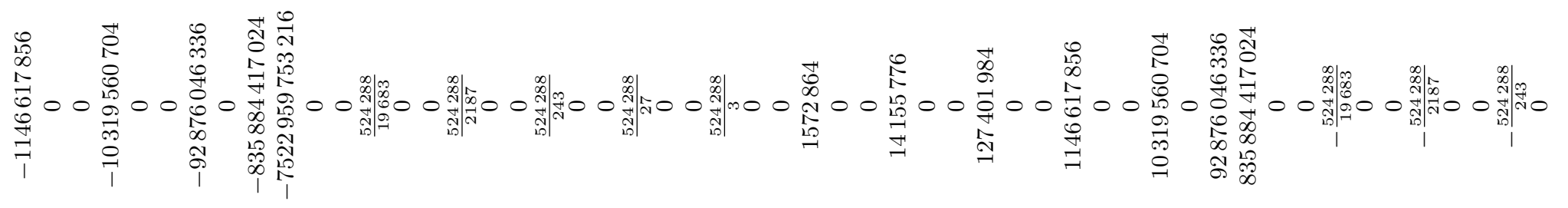

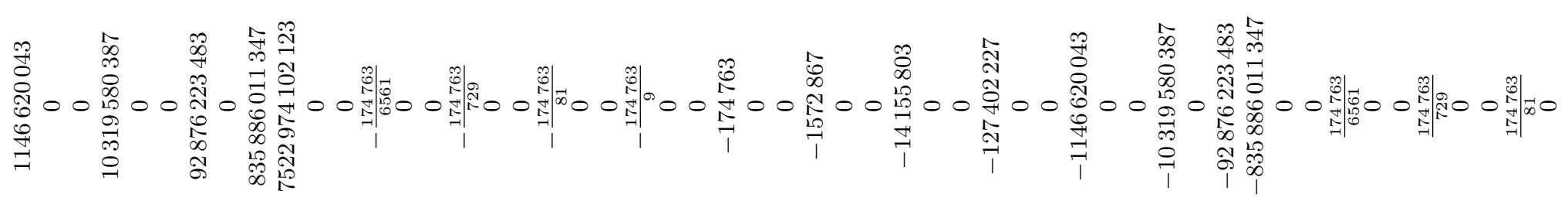

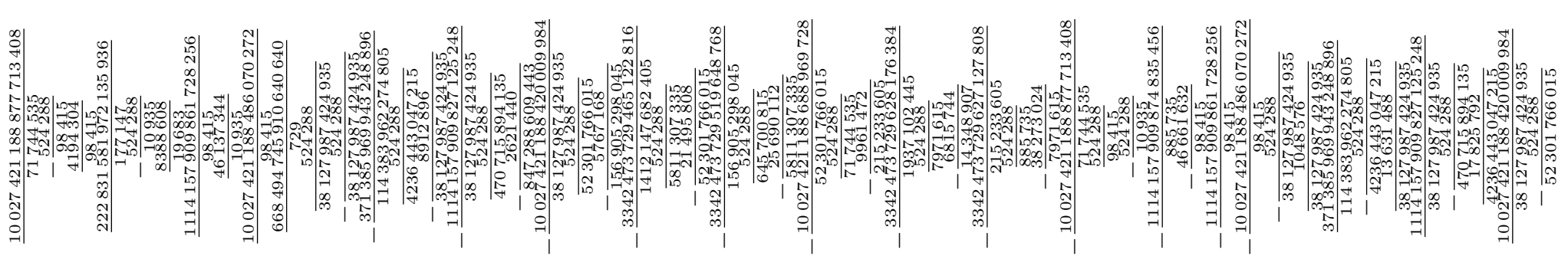

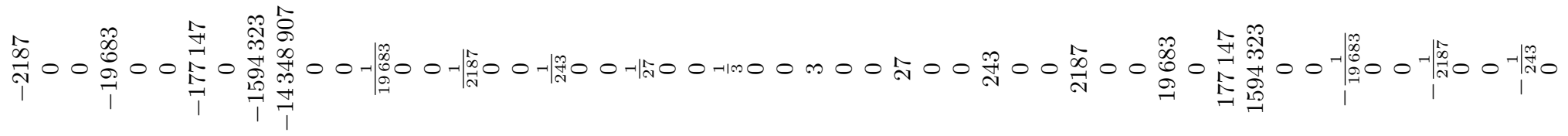
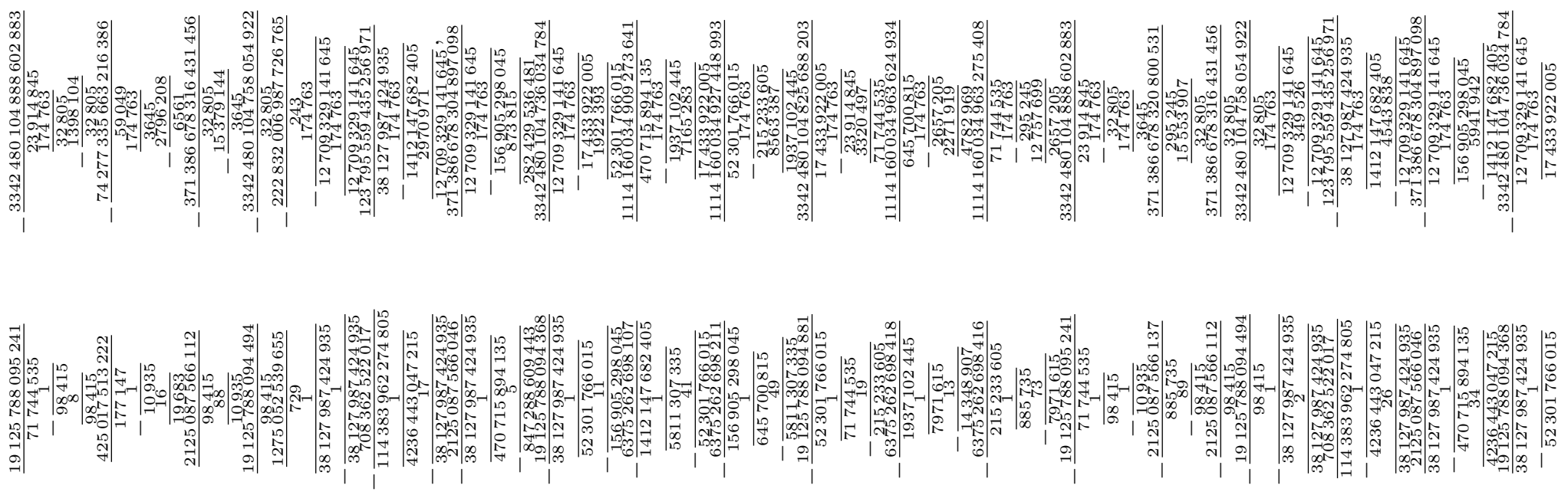

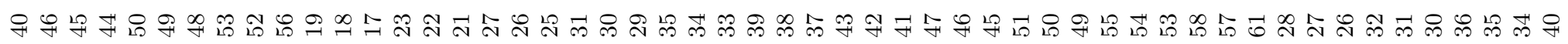

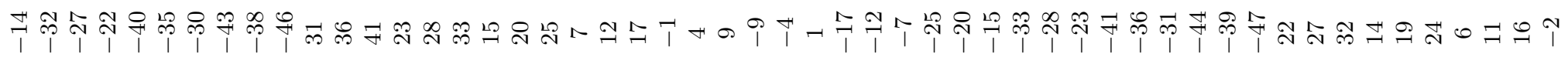

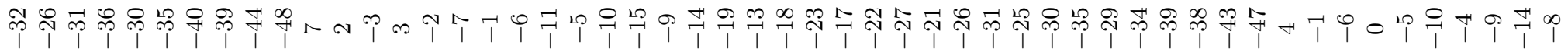

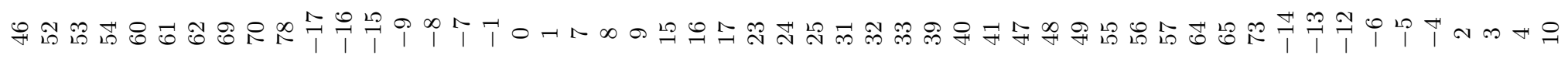

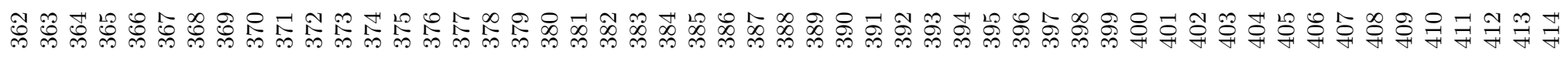




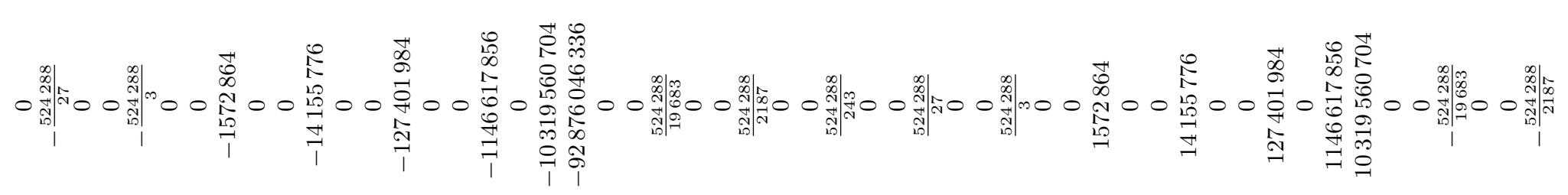

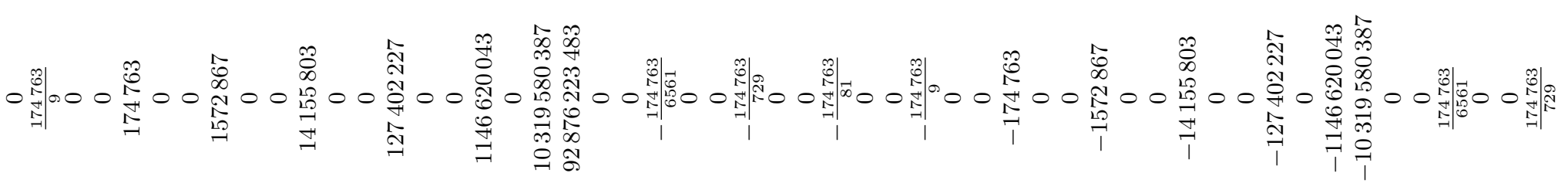

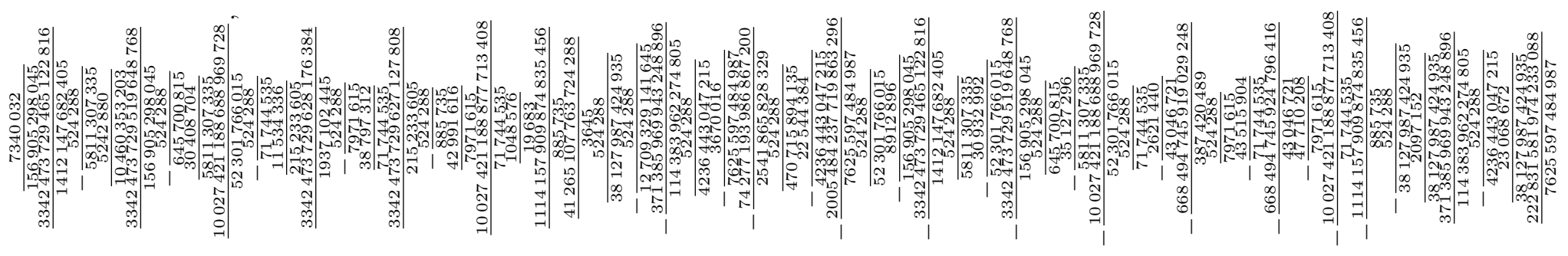

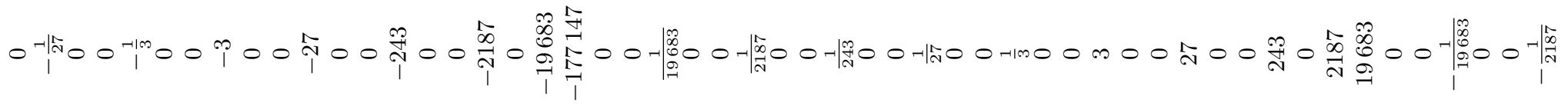
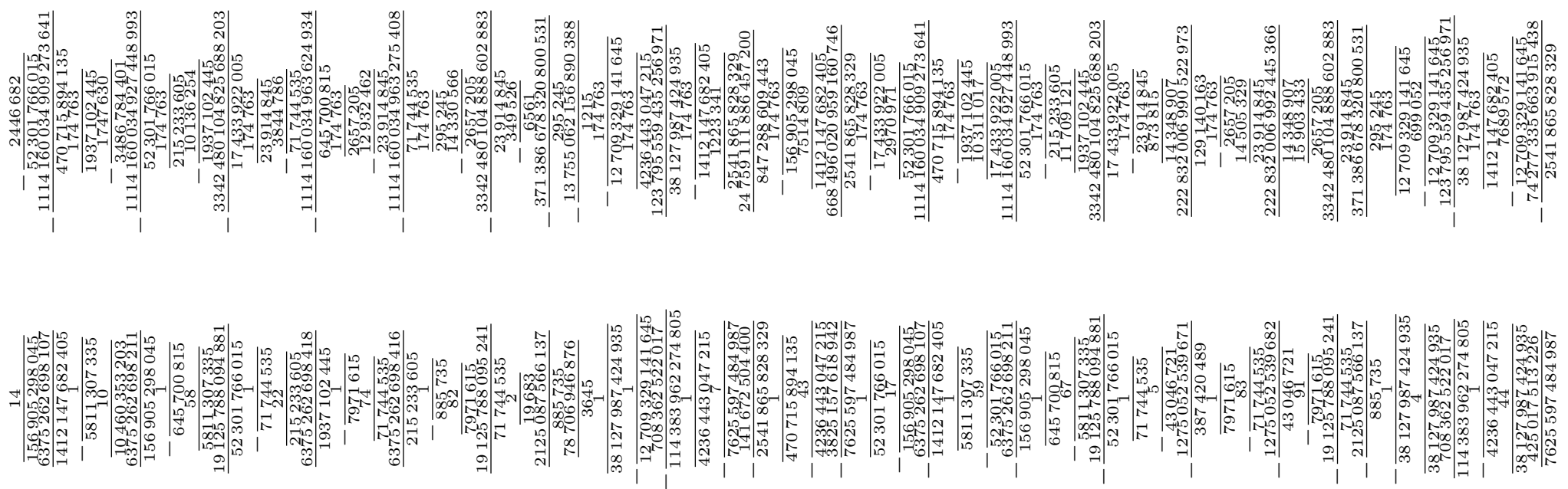

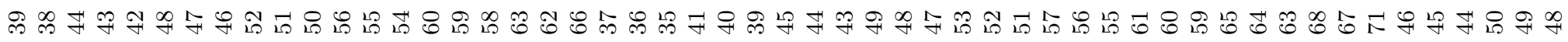
m m

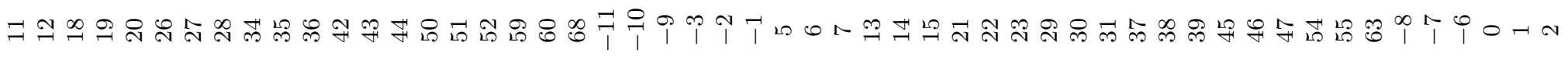

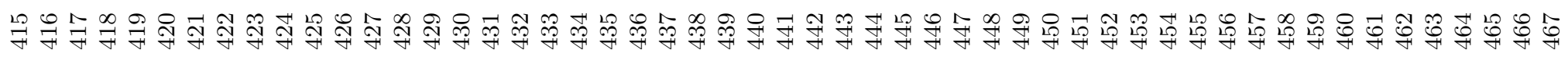




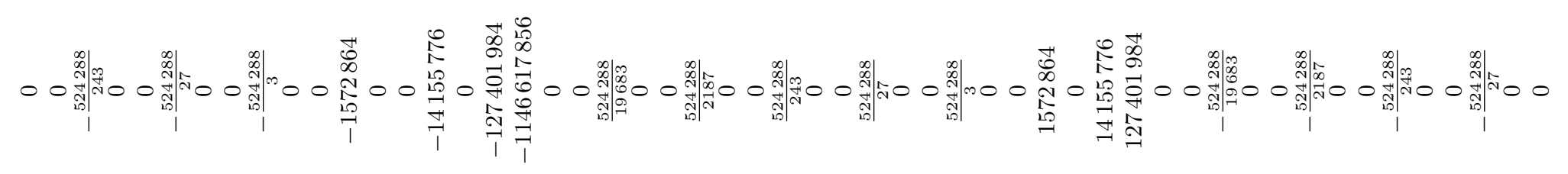
00

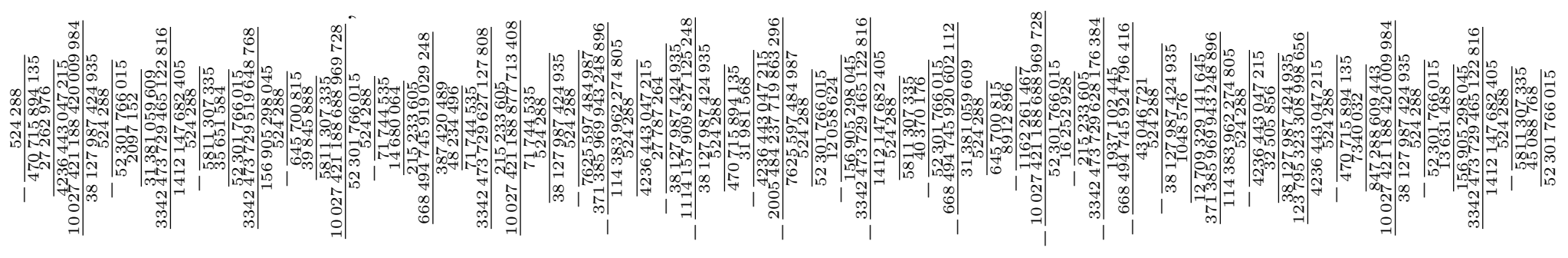

00 悔
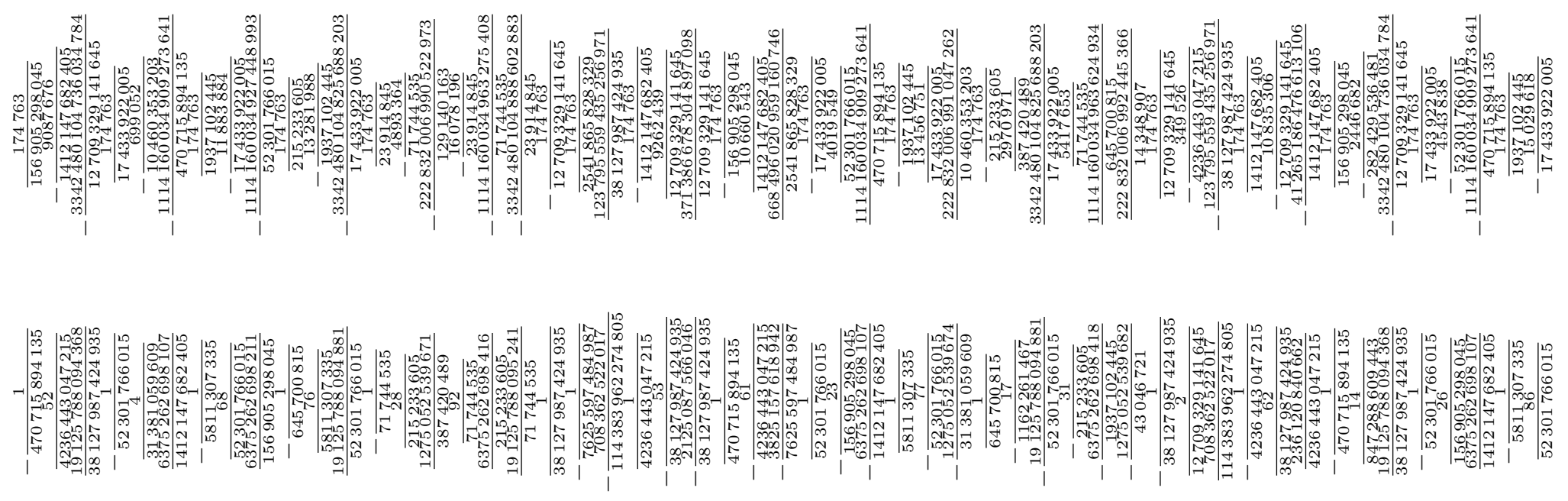

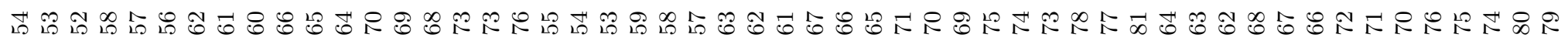

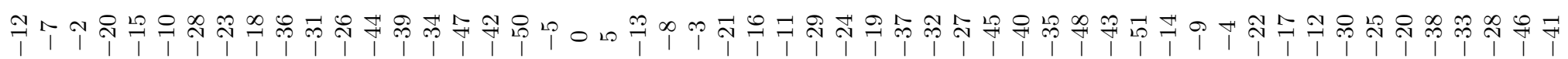

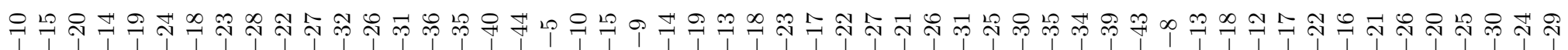

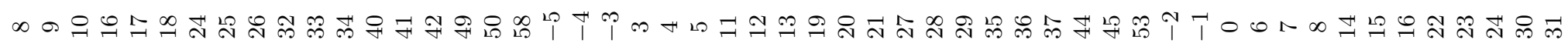

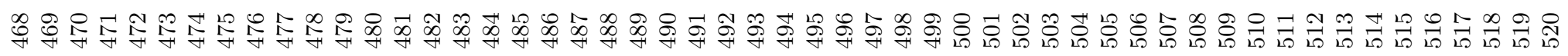




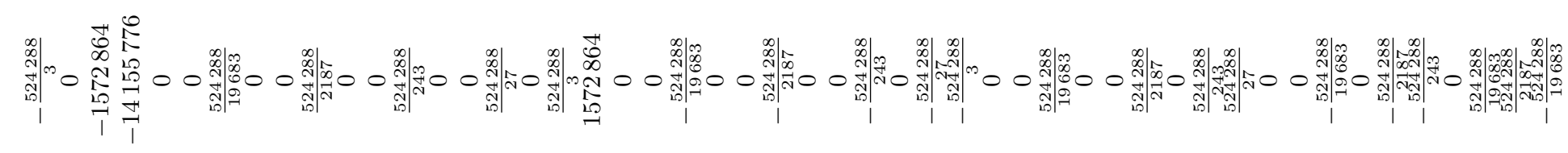

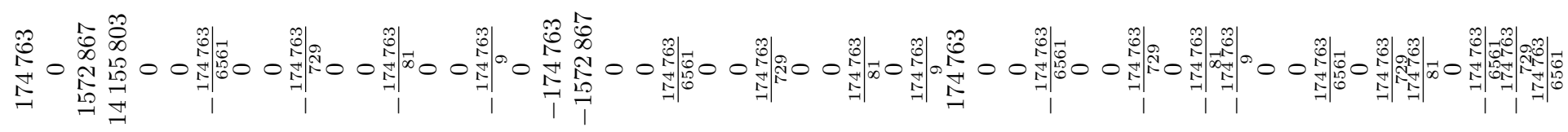

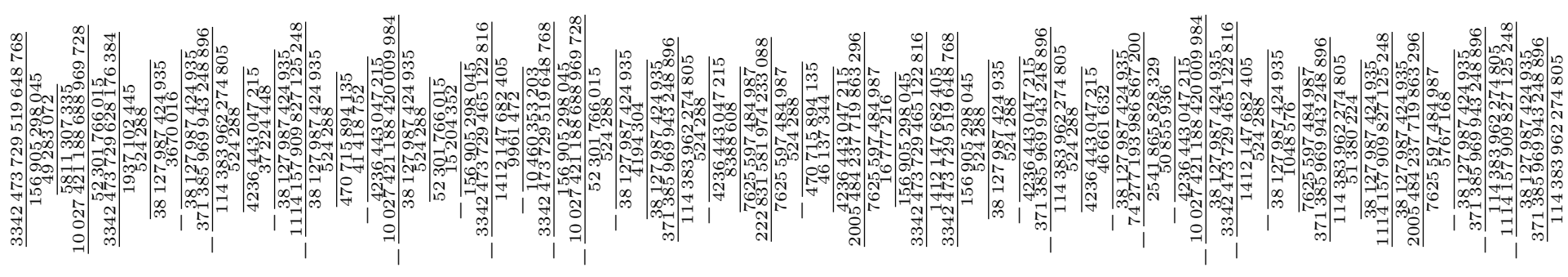

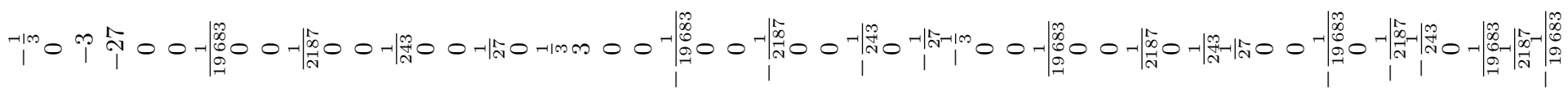
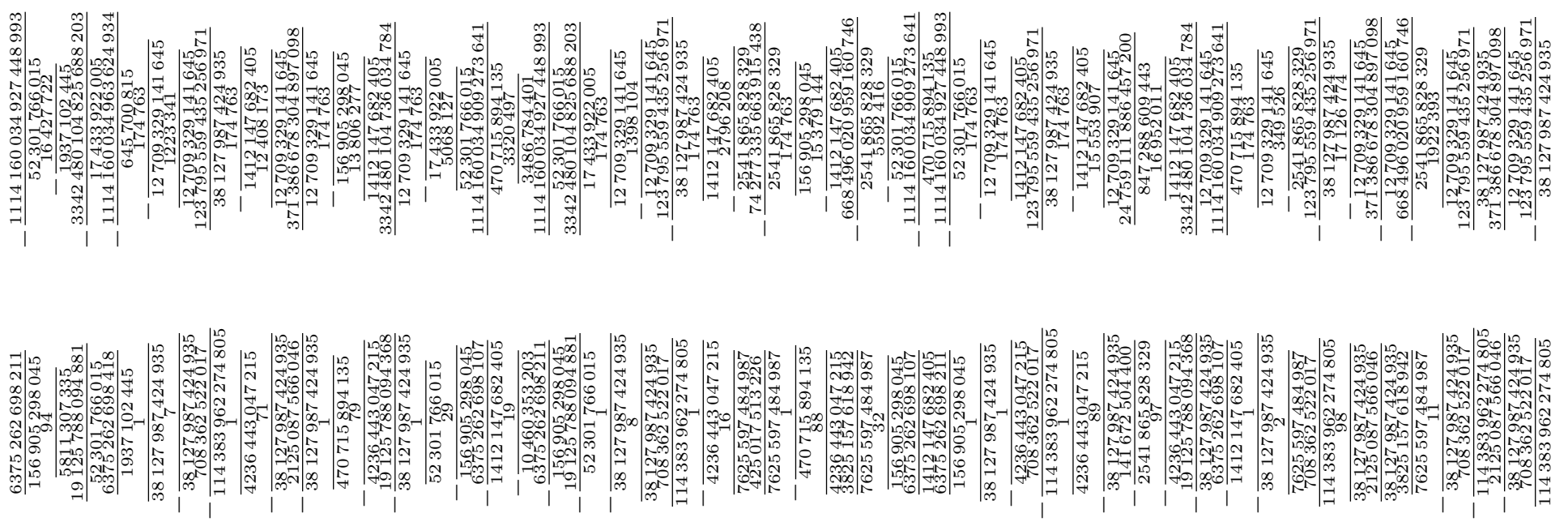

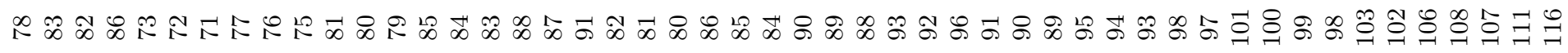

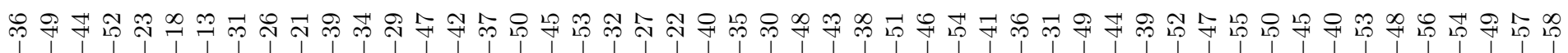

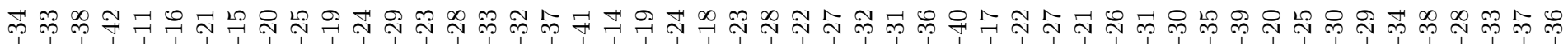

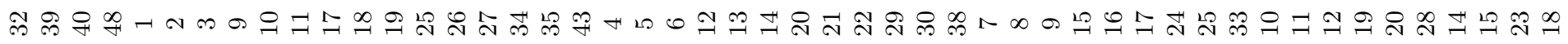

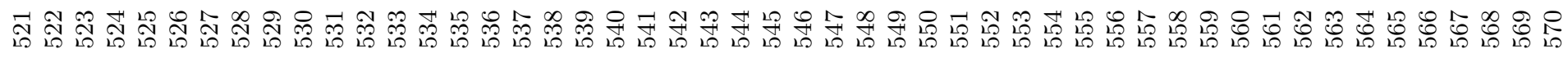




\section{References}

[1] A. Alaca, S. Alaca and K. S. Williams, On the two-dimensional theta functions of Borweins, Acta Arith. 124 (2006), 177-195.

[2] A. Alaca, S. Alaca and K. S. Williams, Evaluation of the convolution sums $\sum_{l+12 m=n} \sigma(l) \sigma(m)$ and $\sum_{3 l+4 m=n} \sigma(l) \sigma(m)$, Adv. Theor. Appl. Math. 1(2006), 27-48.

[3] B. Gordon, Some identities in combinatorial analysis, Quart. J. Math. Oxford Ser. 12 (1961), 285-290.

[4] B. Gordon and S. Robins, Lacunarity of Dedekind $\eta$-products, Glasgow Math. J. 37 (1995), 1-14.

[5] F. Diamond, J. Shurman, A First Course in Modular Forms, Springer Graduate Texts in Mathematics 228, (2005).

[6] V. G. Kac, Infinite-dimensional algebras, Dedekind's $\eta$-function, classical Möbius function and the very strange formula, Adv. Math. 30 (1978), 85-136.

[7] B. Kendirli, Evaluation of Some Convolution Sums by Quasimodular Forms, European Journal of Pure and Applied Mathematics ISSN 13075543 Vol.8., No. 1, (2015), 81-110.

[8] B. Kendirli, Evaluation of Some Convolution Sums and Representation Numbers of Quadratic Forms of Discriminant 135, British Journal of Mathematics and Computer Science, Vol 6/6, (2015), 494-531.

[9] B. Kendirli, Evaluation of Some Convolution Sums and the Representation numbers, Ars Combinatoria Volume CXVI, (2014), 65-91.

[10] B. Kendirli, Cusp Forms in $S_{4}\left(\Gamma_{0}(79)\right)$ and the number of representations of positive integers by some direct sum of binary quadratic forms with discriminant -79,Bulletin of the Korean Mathematical Society, Vol 49/3, (2012), 529-572.

[11] B. Kendirli, Cusp Forms in $S_{4}\left(\Gamma_{0}(47)\right)$ and the number of representations of positive integers by some direct sum of binary quadratic forms with discriminant -47, International Journal of Mathematics and Mathematical Sciences Vol. (2012), Article ID 303492, 10 pages.

[12] B. Kendirli, The Bases of $M_{4}\left(\Gamma_{0}(71)\right), M_{6}\left(\Gamma_{0}(71)\right)$ and the Number of Representations of Integers, Mathematical Problems in Engineering Vol (2013), Article ID 695265, 34 pages

[13] G. Köhler, Eta Products and Theta Series Identities Springer-Verlag, Berlin, (2011).

[14] I. G. Macdonald, Affine root systems and Dedekind's $\eta$-function, Invent. Math. 15 (1972), 91-143.

[15] Olivia X. M. Yao, Ernest X. W. Xia and J. Jin, Explicit Formulas for the Fourier coefficients of a class of eta quotients, International Journal of Number Theory Vol. 9, No. 2 (2013), 487-503.

[16] I. J. Zucker, A systematic way of converting infinite series into infinite products, J. Phys. A 20 (1987) L13-L17.

[17] I. J. Zucker, Further relations amongst infinite series and products:II. The evaluation of three-dimensional lattice sums, J. Phys. A23 (1990), 117-132.

[18] K. S. Williams, Fourier series of a class of eta quotients, Int. J. Number Theory 8 (2012), 993-1004. 